\title{
La coulée continue des aciers. Un exemple de développement technique où l'étroite coopération entre métallurgistes, constructeurs et exploitants a été d'une grande fécondité
}

\section{Partie III : La solidification en machine de coulée continue (CC) ; application à la coulée de quelques familles de nuances d'acier}

\author{
Jean Saleil ${ }^{*}$, a et Jean Le Coze ${ }^{\mathrm{b}}$ \\ Cercle d'Études des Métaux, École Nationale Supérieure des Mines, 42023 Saint-Étienne Cedex 2, France
}

Reçu le 17 septembre 2018 / Accepté le 17 septembre 2018

\begin{abstract}
Résumé. Les processus de solidification à l'œuvre dans les diverses zones de la machine de coulée sont décrits avec leurs conséquences sur la qualité interne du produit. Les principaux moyens additionnels pour améliorer cette qualité sont passés en revue : brassage électromagnétique, réduction en ligne. Les problèmes posés par la coulée des produits de forte section sont examinés. Les comportements spécifiques à la solidification de certaines familles d'acier sont décrits: acier bas carbone pour produits plats minces, aciers inoxydables, aciers à haut carbone pour roulements.
\end{abstract}

Mots clés : structures de solidification / lingotière / refroidissement secondaire / ségrégations / criques internes / brassage électromagnétique / réduction en ligne / aciers bas carbone / aciers inoxydables / aciers pour roulement

\begin{abstract}
Continuous casting of steels. Part III: Solidification in a conti-casting machine; application to some steel grades. Solidification processes in the various zone of the casting machine are described, together with their effects on product internal quality and additional means to improve as cast quality: electro-magnetic stirring and on line reduction. The casting of heavy products is surveyed. Continuous Casting of some specific grades is surveyed in respect to specific required quality: low carbon steels for thin flat products, stainless steel, high carbon steel for bearings (52100 grade).
\end{abstract}

Keywords: solidification structures / mold / secondary cooling / segregation / internal cracks / electromagnetic stirring / in line reduction / low carbon steels / stainless steels / ball bearing steels

\section{Introduction}

Aussi longtemps que les sidérurgistes ont coulé en lingots, ils sont restés assez largement démunis pour agir sur la solidification du produit, ils devaient subir, sans pouvoir vraiment influencer (sauf par la surchauffe), les structures

\footnotetext{
* e-mail: cemetaux@emse.fr

a Ingénieur civil des Mines (Nancy 59), carrière dans les sociétés Ugine (Moutiers), Imphy, Creusot-Loire, Ascometal ; longtemps en charge des développements en matière de procédés d'élaboration.

b Ingénieur civil des Mines (Saint-Étienne 63), Dr. Es Sciences, ex-enseignant chercheur ENSMSE.
}

dites «brutes de coulée». Le passage en coulée continue permettait pour la première fois non seulement d'agir avec une certaine efficacité sur ces processus en localisant dans l'espace les diverses étapes de la solidification (les phénomènes de germination et croissance principalement en lingotière, les phénomènes de ségrégation principalement dans le refroidissement secondaire), mais en outre ce passage en CC exigeait du procédé un pilotage précis pour que la qualité du produit coulé reste compatible avec la simplification des opérations de laminage en aval (suppression du laminoir dégrossisseur), ce qui avait pour conséquence une moindre atténuation des hétérogénéités compositionnelles et structurales issues du processus de solidification. Cette problématique sera à la racine des 
réflexions des aciéries spécialisées dans la coulée des produits de forte section cherchant à passer en coulée continue, nous y consacrerons quelques paragraphes.

\section{Importance de la qualité du métal présenté à la coulée}

Deux caractéristiques du métal en poche de coulée vont fortement influencer les performances qualitatives de la machine de coulée continue:

- la surchauffe du métal liquide à l'entrée de la machine. Une surchauffe trop importante va non seulement altérer les structures de solidification, mais encore accroître le risque de percée par diminution de l'épaisseur solidifiée en lingotière. On admet que la température de l'acier entrant en lingotière ne doit pas dépasser de plus de $5{ }^{\circ} \mathrm{C}$ celle du liquidus de la nuance. On en déduit la température qui doit être visée en poche compte tenu de la chute de température en répartiteur. Cette surchauffe doit être maintenue dans d'étroites limites pendant toute la coulée, ce qui est assez facile pour des poches de fort tonnages, beaucoup moins pour des tonnages limités, (inférieurs à $30 \mathrm{t}$ ). On peut alors interposer un moyen de réchauffage (le plus souvent par induction) entre la poche et la tête de machine;

- la propreté inclusionnaire du métal présenté à la coulée. Une propreté insuffisante peut conduire non seulement à une dégradation des propriétés d'emploi du métal mis en forme [1], mais encore à des perturbations du fonctionnement de la machine, par exemple par colmatage progressif des busettes de coulée par des dépôts inclusionnaires (cas des nuances calmées à l'aluminium et n'ayant pas reçu de traitement au calcium), avec impossibilité de respecter la consigne de vitesse de coulée et même blocage de l'alimentation de la ligne.

Le développement de la métallurgie secondaire en poche à l'aval du convertisseur ou du four électrique de fusion, à partir des années 1970, a fortement contribué à la maîtrise du procédé de coulée continue en améliorant fortement la propreté du métal liquide présenté à la coulée. Il a aussi aidé à sa maîtrise thermique, dans la mesure où le garnissage réfractaire des poches, du fait de la longueur des opérations de métallurgie secondaire, était en bien meilleur état d'équilibre thermique avec le métal liquide contenu, ce qui avait pour conséquence de réduire fortement la chute naturelle de température du métal en poche en cours de coulée.

\section{La solidification en machine: description du processus et techniques de contrôle}

L'annexe A à la fin de cette troisième partie définit quelques notions fondamentales de solidification.

Nous avons déjà signalé que la solidification en lingotière, par la formation de la peau du produit, jouait un rôle déterminant dans la qualité de surface de celui-ci. C'est particulièrement vrai pour les produits plats minces (en aciers au carbone ou en aciers inoxydables). La maîtrise de la solidification dans le refroidissement secondaire est particulièrement importante pour les produits longs, ou les tôles fortes, notamment sur les nuances où les tendances à la ségrégation sont élevées (aciers à forte teneur en carbone).

\subsection{Le faciès de solidification du produit solide en sortie machine}

La solidification est un processus de germination et croissance. C'est de la compétition de ces deux phénomènes, agissant aux divers niveaux de la ligne de coulée (lingotière et refroidissement secondaire), que résultent les structures des produits coulés en continu. Trois facteurs principaux agissent sur la présence des germes de solidification :

- la germination hétérogène, par exemple sur les accidents de la micro-géométrie de la paroi de la lingotière, mais aussi sur des particules solides présentes dans le liquide;

- l'ensemencement du bain liquide par des cristallites arrachées au front de solidification par convection naturelle ou forcée;

- la surchauffe du métal coulé: la germination spontanée (germination homogène) ne se produisant qu'en condition de surfusion importante (température du liquide inférieure au liquidus de la nuance d'acier coulée), elle n'aura qu'un rôle extrêmement limité dans la solidification en machine; en revanche une surchauffe excessive détruira les germes de solidification venus d'ailleurs (germination hétérogène); on en déduit que le bon déroulement de la solidification supposera que le métal liquide présente une surchauffe très limitée à l'entrée dans la lingotière.

Il résulte de ces processus que tout produit issu de coulée continue présente une organisation structurale (dite brute de coulée) offrant un double faciès (Fig. 1):

- à la périphérie une zone dite colonnaire, correspondant à la croissance radiale de cristaux ayant germé à la paroi de la lingotière, qui se fait dans le sens du gradient thermique. Elle s'étend sur plusieurs centimètres à partir de la peau. Elle peut être précédée à l'extrême peau, au contact de la paroi refroidie, d'une zone inframillimétrique de très fine cristallisation qualifiée parfois de «zone de coquille» correspondant à la multiplication des germes à la paroi froide mais qui n'ont pas tous engendré une croissance colonnaire. Cette croissance colonnaire s'interrompt soit parce que le gradient thermique devient insuffisant, soit parce que la température diminuant dans la zone liquide, la germination y devient très active et finit par bloquer la croissance colonnaire, soit parce que la surfusion au front de solidification (Annexe A), principal moteur de la croissance colonnaire devient insuffisante;

- au centre une zone dite équiaxe (sans orientation privilégiée) où les cristaux se sont formés essentiellement par germination sur des particules solides, ou sur des fragments de cristaux colonnaires entraînés par des mouvements de convection naturelle ou forcée. 


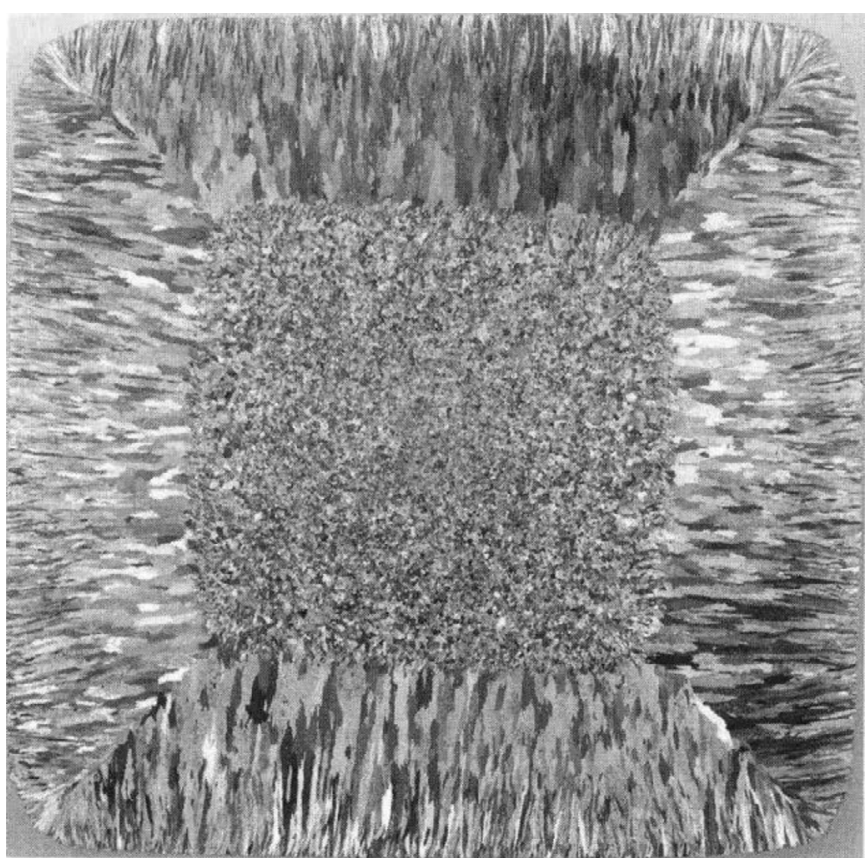

Fig. 1. Structure brute de coulée en sortie machine [2].

Fig. 1. Columnar and equiaxed zones in $C C$ product.

Les proportions respectives des zones colonnaire et équiaxe sur le produit solide brut de coulée dépendent de plusieurs paramètres interdépendants: l'intervalle de solidification de l'acier, le niveau de surchauffe du liquide en lingotière fonction de la température de coulée, l'intensité des refroidissements, les convections naturelles ou forcées (brassage électromagnétique), la vitesse d'extraction du produit et bien sûr le format coulé.

Selon la nuance coulée et en fonction de la gamme de transformation à chaud envisagée pour le produit, on pourra chercher à privilégier la structure équiaxe. Par exemple, sur une nuance présentant une très forte tendance à la ségrégation, une zone colonnaire trop développée peut conduire à des joints colonnaires fortement ségrégés entraînant une fragilité à chaud du produit. Cependant, pour certaines transformations à chaud particulières, notamment pour la mise de ronds en tubes, une structure colonnaire développée peut être un double avantage: le travail de laminage à chaud se fait sur une zone colonnaire de bonne compacité et de bonne pureté de ce fait peu endommageable, les parties ségrégées de la zone équiaxe peu étendue seront tartinées, lors du perçage à chaud, sur l'intrados du tube et n'affecteront que marginalement ses caractéristiques. Mais pour la plupart des produits et des nuances, une zone équiaxe développée contribuera à limiter le phénomène de ségrégation majeure (voir Sect. 3.4) et améliorera la compacité du produit (si l'on a recourt au brassage). On recherchera aussi à obtenir une zone équiaxe dont les grains primaires soient les plus petits possibles; cela suppose d'activer le processus de germination, notamment en limitant la surchauffe et en mettant en œuvre un brassage électromagnétique en haut de machine.
Tableau 1. Effet de la surchauffe sur la structure de solidification, acier à $0,2 \% \mathrm{C}$ [3].

Table 1. Effect of overheating on the solidification structure.

\begin{tabular}{llll}
\hline $\begin{array}{l}\text { Surchauffe } \\
\text { au répartiteur }\end{array}$ & $\begin{array}{l}\text { Zone } \\
\text { basaltique } \\
(\%)\end{array}$ & $\begin{array}{l}\text { Zone } \\
\text { équiaxe }\end{array}$ \\
\hline & & $\begin{array}{l}\text { Structure } \\
\text { grossière } \\
\text { dendritique }\end{array}$ & $\begin{array}{l}\text { Structure fine } \\
\text { non dendritique }\end{array}$ \\
& & $22 \%$ & - \\
$40^{\circ} \mathrm{C}$ & 78 & $44 \%$ & - \\
$30^{\circ} \mathrm{C}$ & 56 & - & $90 \%$ \\
$15^{\circ} \mathrm{C}$ & 10 & & \\
\hline
\end{tabular}

\subsection{La surchauffe du métal à l'entrée de la lingotière : un paramètre essentiel pour le contrôle de la solidification}

Dans la littérature technique, la définition de la surchauffe n'est pas toujours précise. On trouve souvent: TcouléeTliquidus qu'il faut en général interpréter comme la surchauffe au répartiteur. En réalité, c'est la surchauffe en lingotière qui est la variable principale pilotant la solidification. Elle est le résultat des refroidissements cumulés de l'acier liquide: depuis la poche jusqu'au répartiteur, dans les circulations du métal au niveau du répartiteur, dans la busette de coulée. Tous ces transferts thermiques sont maîtrisés grâce à des préchauffages intenses des garnissages et pièces réfractaires au contact de l'acier liquide, avant le démarrage de la séquence de coulées.

Les aciers pour rond à béton, souvent coulés en «jet libre» (c. à d. sans busettes plongeantes) acceptent une surfusion en lingotière pouvant atteindre $20^{\circ} \mathrm{C}$. En revanche, pour des aciers de qualité, la surchauffe visée est nettement plus faible, de quelques degrés dans la lingotière.

Lorsque la surchauffe est élevée, la zone équiaxe se réduit ou disparaît, car les fragments de dendrites qui sédimentent depuis la lingotière se dissolvent dans le liquide et ne peuvent plus servir de germes. Il faut donc abaisser la température au cœur du liquide, par exemple par brassage, pour diminuer la zone colonnaire. Le tableau 1 illustre l'effet de la surchauffe sur l'importance et la finesse de la zone équiaxe.

\subsection{La solidification en lingotière. Brassage électromagnétique en lingotière}

C'est en lingotière que s'amorce et se développe une bonne partie de la zone colonnaire. En avant du front colonnaire se trouve une phase liquide renfermant une proportion plus ou moins grande de cristallites en croissance, et qui s'enrichit en éléments (C, S, P, Mn...) rejetés par le processus de solidification (Annexe A). 


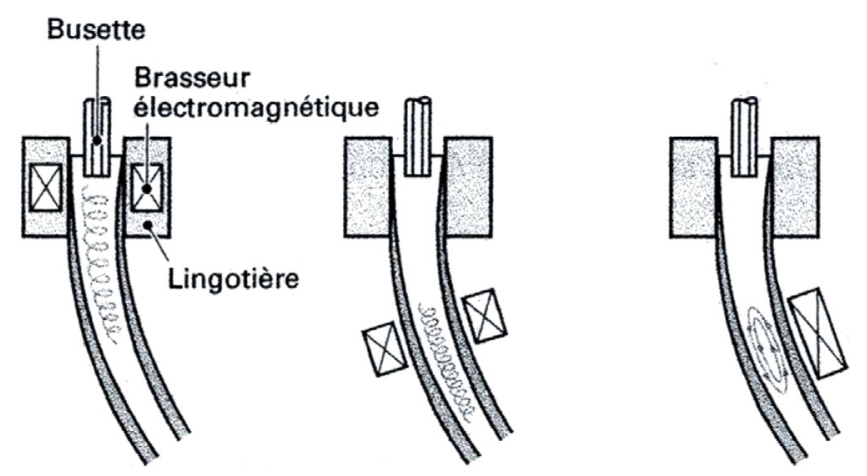

Fig. 2. Brassages électromagnétiques [4]. En lingotière : brassage rotatif (à gauche); dans le secondaire: au centre brassage rotatif, à droite brassage linéaire.

Fig. 2. Electromagnetic stirring.

On conçoit qu'un brassage électromagnétique en lingotière (encore dit brassage primaire) (Fig. 2) soit un moyen de choix pour influencer les structures de solidification. Ce brassage agit selon deux mécanismes: (1) en arrachant au front de solidification les cristallites qui vont être autant de germes pour la zone équiaxe, il va favoriser la formation de celle-ci; (2) en induisant un flux transversal de métal chaud au front de solidification, il peut interrompre la croissance des grains basaltiques. La position du brasseur sur la ligne de coulée (rotatif pour les produits longs ; linéaire pour les produits plats) : au bas de la lingotière ou immédiatement sous la lingotière, ainsi que l'intensité du brassage appliqué, auront un effet déterminant sur le résultat de l'opération. Le mieux peut être l'ennemi du bien avec l'apparition d'un cerne de ségrégation négative au front de solidification (par lavage $\mathrm{du}$ front colonnaire) lorsque celui-ci entre dans la zone d'influence du brasseur. Dans le cas de la coulée avec poudre sur le ménisque, un brassage trop intense, ou trop proche du ménisque peut provoquer des pollutions inclusionnaires par entraînement de particules arrachées au laitier de coulée.

Le dispositif d'alimentation en métal chaud, par les circulations induites en lingotières, a une influence certaine non seulement sur la solidification en lingotière, mais encore plus bas dans le puits liquide, notamment dans le cas de la coulée des brames. Les busettes à ouies et le frein électromagnétique sont des moyens d'agir sur la distribution, au sein de la lingotière, du métal chaud issu du répartiteur et d'éviter que des flux massiques et thermiques mal répartis perturbent trop les structures de solidification.

Pour l'histoire, on notera que le brassage électromagnétique est déjà mentionné dans les brevets Junghans et appliqué dès 1952 sur la machine pilote de Mannesmann Hückinghen démarrée en 1950.

\subsection{La solidification dans le refroidissement secondaire. La ségrégation majeure et son contrôle}

La manière dont cette solidification à cœur du produit coulé sera plus ou moins contrôlée va influencer fortement sa santé interne.
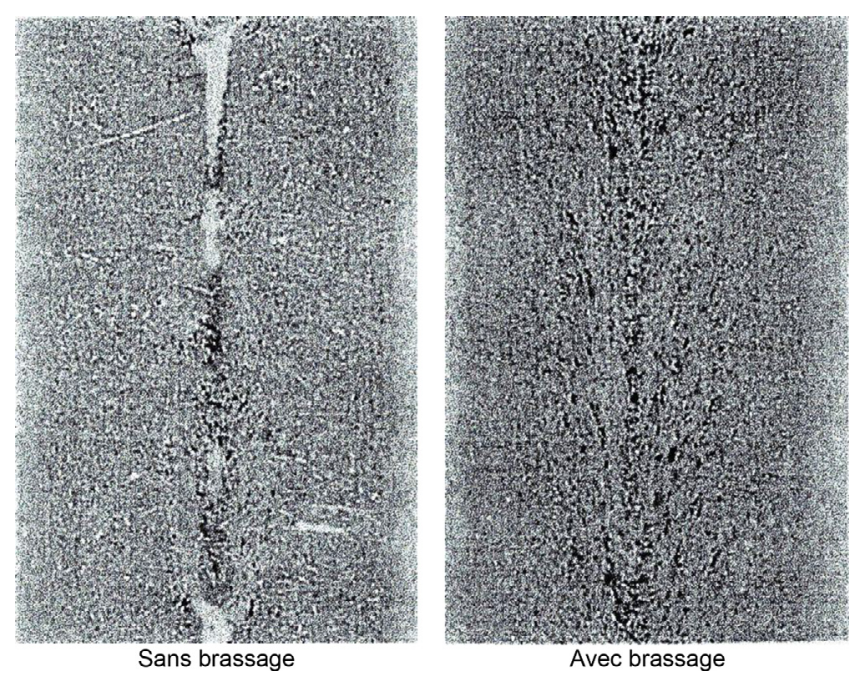

Fig. 3. Macrostructures internes de solidification avec et sans brassage [4].

Fig. 3. Solidification macrostructures with and without stirring.

\subsubsection{Solidification à cœur du produit}

$\mathrm{Au}$ long de la ligne de coulée, le cour du produit est constitué d'un mélange de phase liquide et de cristallites qui peut localement être décrit par deux caractéristiques : la fraction liquide et la température.

La fraction liquide croît de la peau vers le cœur et décroît à mesure que le produit progresse dans la ligne de coulée en s'éloignant de la lingotière. La partie de cette zone centrale de solidification au contact du front colonnaire, où la fraction liquide est faible, est qualifiée de zone pâteuse (Annexe A).

La température croît de la peau vers le cœur et décroît à mesure que le produit avance dans la ligne de coulée, tout en restant à l'intérieur de l'intervalle de solidification défini par la composition locale.

La figure 3 est une représentation schématique de telles structures de solidification avec et sans brassage électromagnétique. On observe que le brassage a eu pour effet d'effacer les vides périodiques axiaux (retassures) et de disperser les zones ségrégées. La figure 4 schématise l'évolution des fractions liquides au long de la ligne de coulée.

À mesure que la solidification progresse, la phase liquide s'enrichit en éléments rejetés par le processus de solidification. C'est en particulier le cas pour les éléments C, S, P, $\mathrm{Mn}$. On qualifie ce processus de ségrégation majeure. En l'absence de toute action physique pour contrer ce phénomène, les écarts de compositions locales entre cœur et peau peuvent être suffisamment importants pour affecter localement soit l'aptitude à la transformation à chaud du produit, soit les propriétés d'emploi du matériau. Ils sont donc une cause majeure de l'hétérogénéité et de l'anisotropie structurale du matériau et à ce titre ils ont été les freins principaux au développement de la CC.

Ce phénomène de ségrégation majeure croît avec l'épaisseur du produit coulé mais est atténué si la zone équiaxe est suffisamment développée, ce qui suppose au minimum une faible surchauffe. 


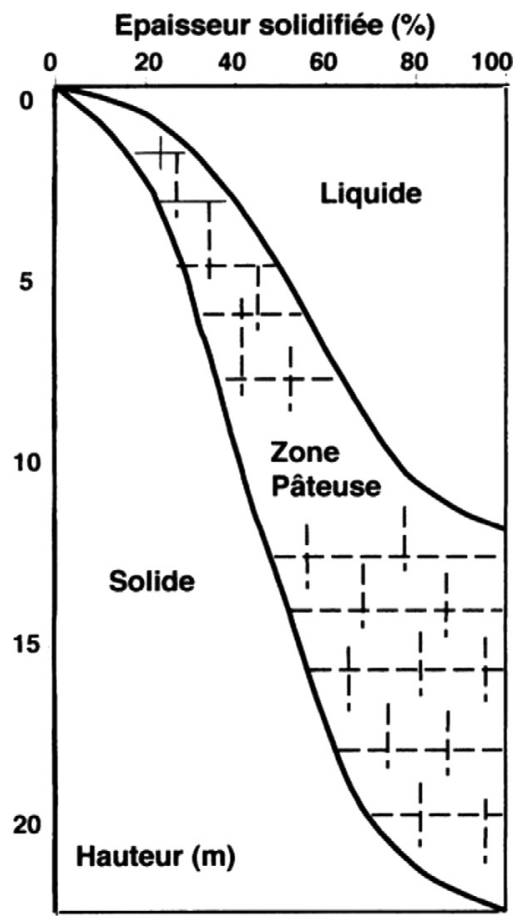

Fig. 4. Schéma d'évolution des fractions liquides au long de la ligne, d'après [4]. À $12 \mathrm{~m}$ sous le ménisque, il n'y a plus de liquide, mais la zone pâteuse ne se ferme qu'au-delà de $20 \mathrm{~m}$.

Fig. 4. Liquid fraction variation along the solidification line.

Le tableau 2 illustre l'effet de la surchauffe sur l'étendue de la zone équiaxe et les taux de ségrégation en $\mathrm{S}$ et Mn sur un acier à $0,12 \% \mathrm{C}$ et $0,60 \% \mathrm{Mn}$.

\subsubsection{Mini retassures et gonflements}

La ségrégation centrale se présente selon un faciès différent selon qu'on considère des ébauches destinées aux produits longs (blooms et billettes) ou aux produits plats (brames). La figure 5 schématise la répartition des ségrégations dans une brame ou un bloom:

- dans les produits longs, la fermeture du puits liquide se traduit par une macro-structure périodique appelée "mini-lingot" (Fig. 6), qui renforce la ségrégation centrale, associée à des mini retassures (contraction à la solidification);

- dans les produits plats, en plus du phénomène de minilingot, on a affaire à de possibles gonflements de la brame qui entraînent un flux de métal liquide riche en solutés, c'est-à-dire une ségrégation par aspiration de métal vers les fronts pâteux parallèles aux deux grandes faces.

L'augmentation de l'intensité du refroidissement secondaire joue sur la ségrégation centrale:

- par l'obtention d'une peau plus rigide, elle diminue les risques de gonflement et donc ceux d'apparition de veines ou criques ségrégées correspondantes.

- par raccourcissement du puits liquide [9], elle diminue la pression hydrostatique à l'origine des gonflements.
Nous avons vu en Partie II que c'était essentiellement la qualité du supportage qui limitait, sur les machines à brames, le phénomène de gonflement et les ségrégations associées [26].

Cette zone de solidification complexe au centre du produit, où coexistent solide et liquide à la composition enrichie en éléments ségrégeants (donc à solidus fortement abaissé) et aux édifices solides fragiles, qui selon l'épaisseur des produits coulés peut s'étendre en longueur sur plus d'une dizaine de mètres, peut être soumise à de nombreux phénomènes perturbateurs affectant les structures pâteuses ou solides en cours de mise en place:

- actions mécaniques extérieures subies (désalignement des rouleaux de soutien, gonflement, décintrage...);

- actions extérieures volontaires (brassage électromagnétique secondaire, réduction mécanique);

- actions des phénomènes physico-chimiques liés à la progression de la solidification (convection thermique, convection solutale, convection liée aux écarts de densité...).

Ces phénomènes peuvent d'autant plus imposer leurs effets (généralement sous forme de défauts à cœur du produit: criques internes, porosités, veines ségrégées), qu'ils ont plus d'espace et de temps pour se manifester, notamment sur les produits de très forte section, et que la nuance coulée présentera un large intervalle de solidification (écart de température entre liquidus et solidus) favorisant la mise en place des ségrégations (cas des nuances à teneur élevée en carbone).

\subsubsection{Criques internes}

Les phénomènes physiques précédemment évoqués peuvent conduire à des fissures qui se forment à l'intérieur de la zone pâteuse, dans un solide à température élevée dont la résistance à la déformation est faible. Elles peuvent aussi résulter d'un réchauffage mal maîtrisé de la peau en fin de refroidissement secondaire (lorsque celui-ci s'interrompt), qui entraîne la mise en traction du cœur et la formation de criques centrales en forme d'étoiles, etc. Les criques internes peuvent s'amorcer sur les microporosités ou microretassures formées dans la zone pâteuse quand le liquide résiduel, emprisonné dans le réseau de dendrites, se solidifie en se contractant. Les criques internes s'accompagnent de ségrégation dans les fissures par appel de liquide riche en solutés venant de l'intérieur de la zone pâteuse. Le mouvement du liquide va pouvoir cicatriser les fissures, mais, au fond du puits liquide, la circulation du liquide est quasi nulle et le risque de crique augmente. La réduction en ligne visera à traiter ces problèmes, nous allons y revenir plus en détail.

\subsubsection{Le brassage électromagnétique dit secondaire}

Positionné nettement en dessous de la lingotière, généralement linéaire, il peut être un moyen de lutter contre la mise en place des structures de solidification déficientes, liées aux phénomènes physiques précédemment évoqués, en homogénéisant la composition du puits liquide [6]. Il ne peut être efficace que sur des fractions liquides assez 
Tableau 2. Effet de la surchauffe sur l'étendue de la zone équiaxe et sur les taux de ségrégation (rapport entre la teneur au centre du produit et la valeur nominale) en S et Mn, acier X60 [5].

Table 2. Influence of overheating on equiaxe zone size and segregation of $S$ and $M n$.

\begin{tabular}{llll}
\hline Zone équiaxe (\%) & Ségrégation S\% & Segrégation Mn \% & Surchauffe $^{\mathrm{a}}\left({ }^{\circ} \mathrm{C}\right)$ \\
\hline 15 & 60 & 8 & 20 \\
35 & 16 & 0 & 5 \\
40 & 5 & -2 & $2 \mathrm{f}$ \\
\hline
\end{tabular}

${ }^{\mathrm{a}} \mathrm{T}$ (répartiteur) - $\mathrm{T}$ (liquidus).

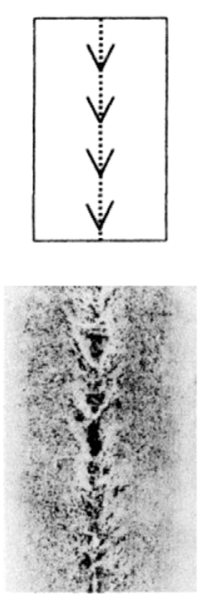

A
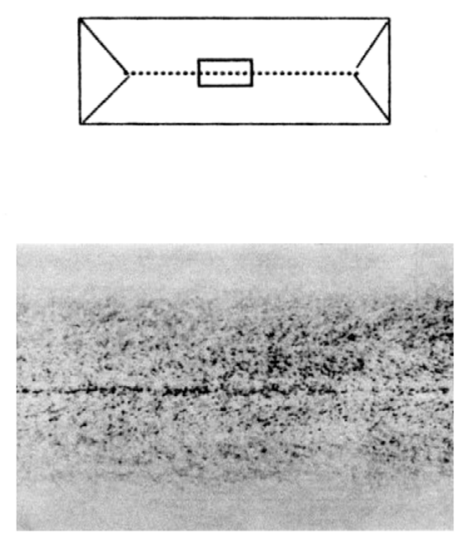

B

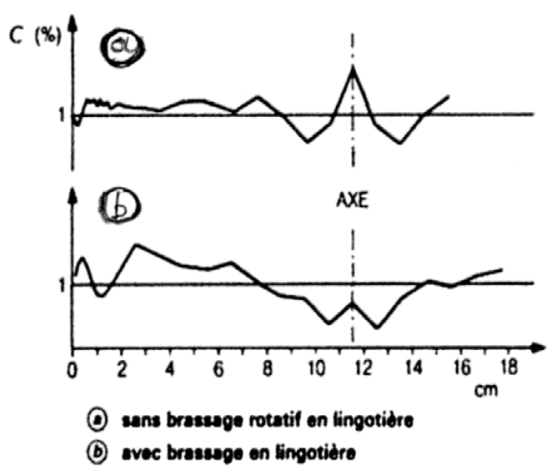

C

Fig. 5. Schéma des ségrégations centrales sur produits de CC. A : coupe verticale sur un bloom:Vés de ségrégation [6]. B : coupe horizontale au centre d'une brame [7]. C: profil de carbone le long d'un rayon d'une billette de 100Cr6, coulée en continu: effet bénéfique du brassage sur la ségrégation axiale (zone à ségrégation négative) [8].

Fig. 5. Central segregation in continuous casting products.

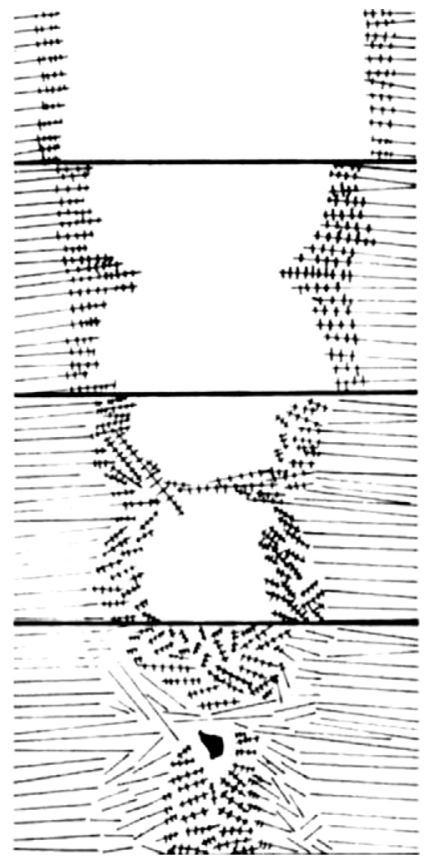

Fig. 6. Structure mini-lingot [7].

Fig. 6. "Mini-ingot" structure.

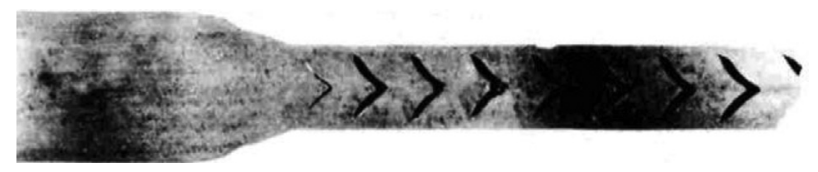

Fig. 7. Chevrons : conséquences observées après mise en forme à froid [6].

Fig. 7. Consequence of V-solidification segregation after cold forming.

élevées, donc à condition d'être positionné pas trop bas sur les lignes de coulée. On notera que le brassage électromagnétique secondaire supprime la formation des chevrons issus des «mini-lingots » (Fig. 7).

Il reste donc à traiter spécifiquement le fond de puits du point de vue de la ségrégation majeure et des divers défauts engendrés par la solidification finissante.

\subsubsection{La réduction en ligne}

Le traitement $\mathrm{du}$ fond de puits liquide, pour maîtriser les défauts de fin de solidification, relève des techniques de réduction en ligne. Elles consistent à exercer sur le produit, dans une zone de la machine judicieusement positionnée, une contrainte mécanique soigneusement dosée (pour éviter de 


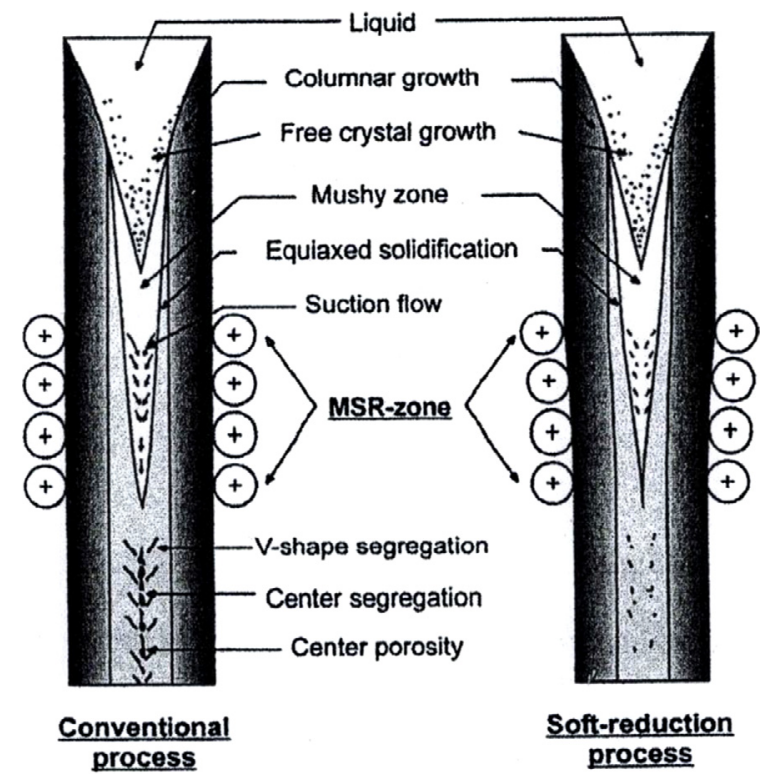

Fig. 8. Influence de la réduction douce sur les structures de solidification [10].

Fig. 8. Influence of soft reduction process on solidification.

déchirer en interne le produit) qui aura pour but de refermer canaux, fissures et porosités en expulsant vers le haut les liquides ségrégés qui y sont contenus. La meilleure image que l'on puisse donner d'un tel processus est celle de l'éponge gorgée de liquide ségrégé que l'on presse délicatement pour en extraire le liquide. On distingue plusieurs degrés dans cette technique de réduction:

- la réduction douce qui peut être soit mécanique (MSR : Mechanical Soft Reduction), soit thermique en appliquant sur une portion du secondaire un refroidissement intense qui va contracter la peau et mettre ainsi le cœur du produit en compression. Il conviendra cependant d'être très attentif au réchauffement de la peau en aval de cette réduction thermique, car il peut induire des criques centrales par dilatation de la peau et mise en traction du cœur du produit. La réduction douce mécanique (MSR) initialement appliquée à la coulée des brames a aussi été étendue aux produits longs. Les contraintes de compression sont appliquées par des cages spécifiques de rouleaux positionnées sur les lignes de coulée généralement en amont des cages extractrices, afin d'agir sur un produit qui doit présenter à cœur une fraction solide comprise entre d'étroites limites. La figure 8 illustre l'effet de la réduction douce sur les structures de solidification. La figure 9 définit schématiquement la zone optimale d'application de la réduction en fonction de la fraction solide au centre du produit, et les limites de l'effort à appliquer pour éviter la formation de criques internes. La figure 10 met en évidence les défauts produits par une réduction douce mal appliquée, sur la billette coulée et sur le fil machine qui en est issu.

La réduction douce pour être pleinement efficace doit s'appliquer en fond de puits sur une fraction liquide inférieure à 0,4 ; son ampleur doit compenser le retrait correspondant à la solidification de cette fraction liquide ; la

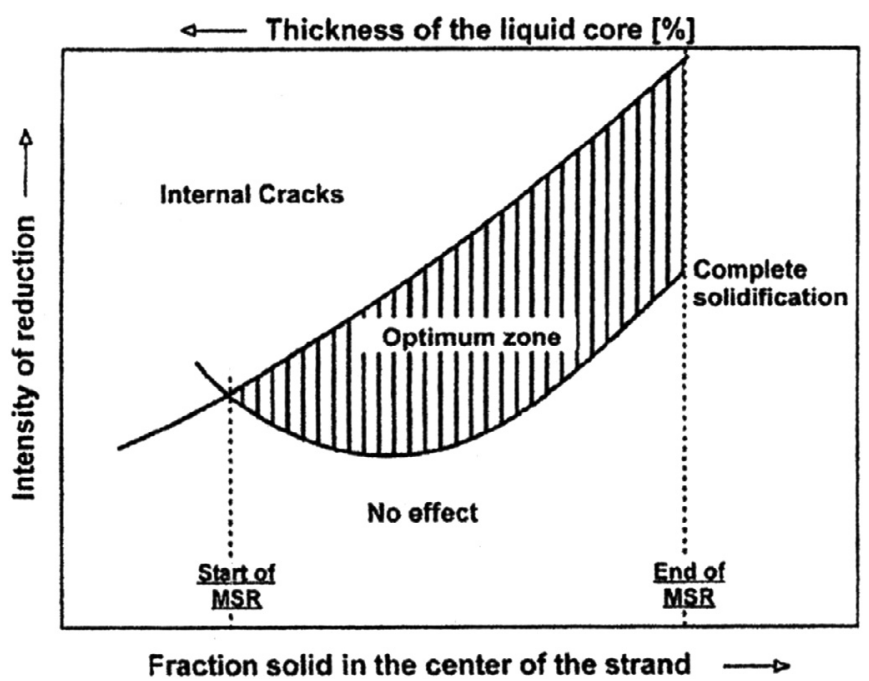

Fig. 9. Domaine opératoire de la réduction douce en fonction de la fraction solide à cœur [11].

Fig. 9. Operational range of soft reduction.

vitesse de la déformation doit correspondre à la vitesse de solidification du fond de puits [12]. On conçoit que l'application de la technique soit assez délicate, qu'elle doive être adaptée à la nuance coulée pour tenir compte du diagramme de phase correspondant à la composition $\mathrm{du}$ fond de puis liquide, et qu'il soit assez facile d'introduire des défauts supplémentaires par un procédé mal réglé (Fig. 10) [11]. Les derniers développements, pour lutter contre les désordres structuraux produits par une application inadéquate de la réduction mécanique, ont consisté à piloter cette réduction d'après une modélisation numérique de l'avancement de la solidification. C'est la Dynamic Mechanical Soft Réduction (DMSR) développée par le constructeur SMS Concast A.G. (voir dans la Sect. 6 la description de la machine de Timken/Faircrest) ;

- la réduction forte ou Mechanical Hard Réduction (MHR), uniquement mécanique, mise en oeuvre sur les coulées de blooms ou de brames de forte épaisseur. La déformation est beaucoup plus importante (plusieurs dizaines de $\mathrm{mm}$ ) que dans le cas de la réduction douce aussi le procédé s'apparente-t-il, dans ses versions les plus extrêmes, à une ou plusieurs passes de forgeage. C'est cette technologie qui s'impose pour la coulée des nuances à haut carbone en blooms de forte section (voir Sect. 6). La figure 11 rend compte de l'application de la MHR sur la machine à blooms (verticale/courbe, format $300 \times 430 \mathrm{~mm}^{2}$ ) $\mathrm{n}^{\mathrm{O}} 3$ de Kobé Steel/Kobé et illustre les effets sur les fractions solides à cour des passes successives de réduction. On notera que la réduction forte, par l'ampleur des déformations à appliquer depuis la peau, repose le problème, bien connu des forgerons, de la ductilité à chaud des nuances considérées et du risque d'apparition de criques de forgeabilité.

Une modalité particulière de la réduction en ligne a été mise en œuvre par Nippon Steel dans le cas des brames. Il s'agit du procédé RIB (Réduction of Intentional Bulging) 

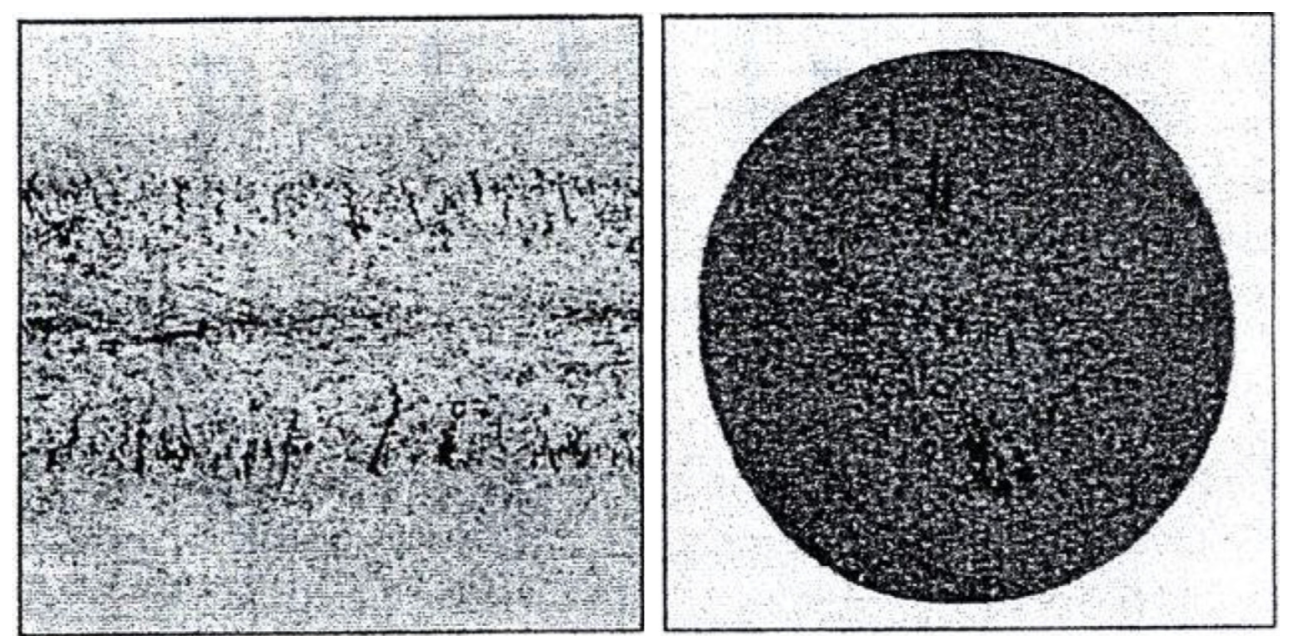

Fig. 10. Effet d'une réduction douce incorrectement appliquée [11]. Image de gauche: sur billette, déchirures au front de solidification ; image de droite : sur fil de diamètre $5,5 \mathrm{~mm}$ laminé à partir d'une billette défaillante (on observe des pôles ségrégés à mirayon).

Fig. 10. Effect of a badly applied soft reduction; left on a billet; right: result on a $\Phi 5.5$ mm rolled wire.

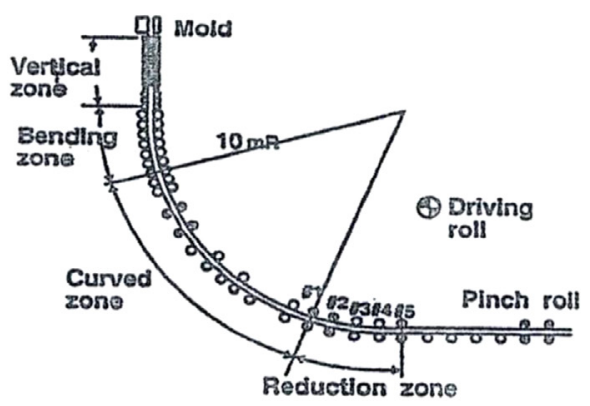

\begin{tabular}{|c|c|c|}
\hline \multicolumn{2}{|c|}{ Ilam } & Specticastions \\
\hline \multicolumn{2}{|c|}{ Machino typo } & Vertical bonding \\
\hline \multicolumn{2}{|c|}{ Bloom atzo } & $300 \times 430 \mathrm{~mm}^{2}$ \\
\hline \multicolumn{2}{|c|}{ Radlus of curvaturo } & $10 \mathrm{~m}$ \\
\hline \multicolumn{2}{|c|}{ ELAS } & LAF ENS \\
\hline \multicolumn{2}{|c|}{ Aaschins langth } & $32.3 \mathrm{~m}$ \\
\hline \multirow{4}{*}{$\begin{array}{c}\text { Roduction } \\
\text { zona }\end{array}$} & ol pressuro & Bax $170 \mathrm{~kg} / \mathrm{cm}^{2}$ \\
\hline & Rell thanoter| & $400 \mathrm{~mm}$ \\
\hline & Rols pltech & $8,200 \mathrm{~mm}$ \\
\hline & Zono langth & $4,800 \mathrm{~mm}$ \\
\hline
\end{tabular}

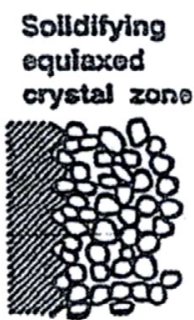

1 si reduetion

2 no

reciuction

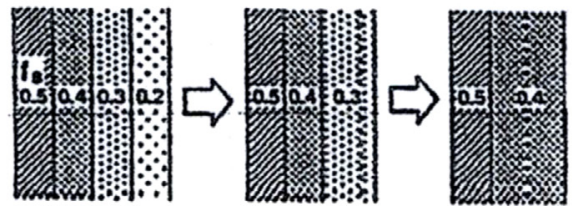

Fig. 11. Effet sur les fractions solides à cœur de deux passes de MHR, d'après [12]. Avant la première réduction, la fraction solide (fs) à cœur est de 0,2. Après la deuxième réduction, elle est de 0,4 au centre de la zone équiaxe.

Fig. 11. Central solid fractions after two MHR reductions.

qui consiste à laisser le gonflement se développer pour le réduire ensuite par un écrasement contrôlé. Ce procédé apparemment paradoxal mérite un éclairage plus précis:

- le gonflement apporte au cœur du produit, en fond de puits, du métal plus chaud et moins ségrégé, ce qui va atténuer l'intensité des ségrégations initiales;

- cet apport de métal liquide va contribuer à combler les porosités ;

- la compression ultérieure se faisant sur une zone à cœur réchauffée, aura moins tendance à endommager le front de solidification.

\subsubsection{Les moyens d'application de la réduction mécanique}

Les efforts à transmettre croissent fortement avec la taille des produits coulés, les constructeurs de machines de coulée ont donc imaginé divers dispositifs, adaptés aux formats coulés (brames ou blooms) pour appliquer judicieusement ces efforts, tout en les minimisant:

- rouleaux de forme (crown roll, shape roll) (Fig. 12);

- rouleaux divisés (Fig. 13) pour moduler le serrage sur la largeur du produit;

- forgeage en ligne (crater end forging) (Fig. 14). 


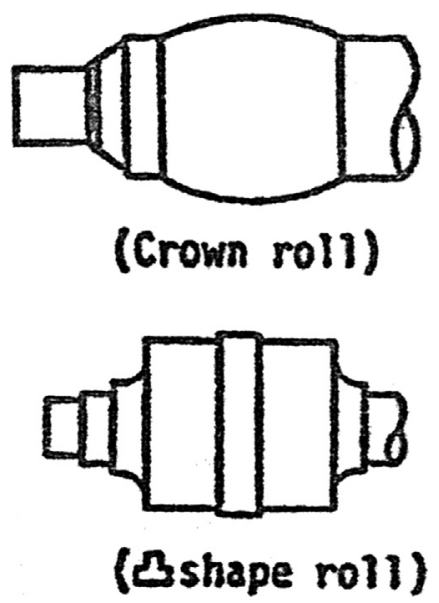

Fig. 12. Rouleaux de forme [12].

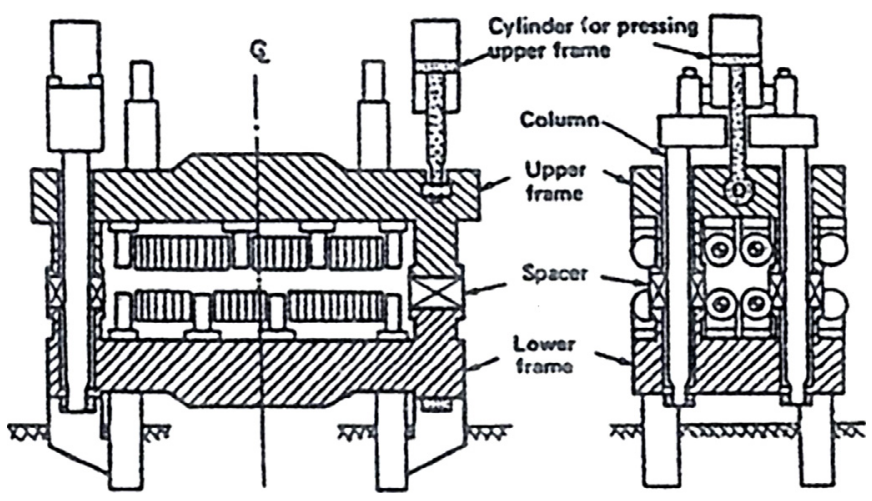

Fig. 13. Rouleaux divisés (avec application de la contrainte de compression) [12].

Fig. 13. Divided rolls for applying soft reduction on slabs.

Fig. 12. Crown roll, shape roll.

\begin{tabular}{|c|c|}
\hline $\begin{array}{l}\text { Ylachine type } \\
\text { Casting radias } \\
\text { Eandish capasity } \\
\text { Bloom size } \\
\text { Casting speed } \\
\end{array}$ & 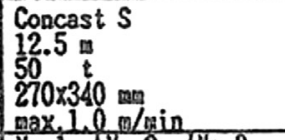 \\
\hline $\begin{array}{l}\text { Distance from meniscus } \\
\text { Type } \\
\text { Brequency }\end{array}$ & \begin{tabular}{|l|l|l|}
$\mathrm{Ho.1}$ & $\mathrm{H} .2$ & $\mathrm{Ko} .3$ \\
$3.6 \mathrm{E}$ & $9.0 \mathrm{~B}$ & $12.0 \mathrm{y}$ \\
Linear & Botary & Rotary \\
$\mathrm{Hz}$ & $12 \mathrm{~Hz}$ & $12 \mathrm{~Hz}$ \\
\end{tabular} \\
\hline $\begin{array}{l}\text { Porging } \\
\text { Distance fros meniscus } \\
\text { Redaction tickness }\end{array}$ & 16 max.100 mm \\
\hline $\begin{array}{l}\text { Chemical composition of } \\
\text { C: } 0.05 \sim 1.00,51: 0.20\end{array}$ & $\begin{array}{l}\text { steel }(\%) \\
\sim 0.70, \mathrm{Hn}: 0\end{array}$ \\
\hline
\end{tabular}

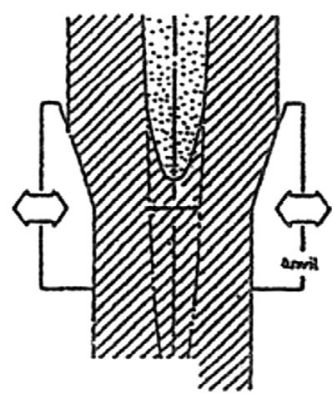

Fig. 14. Forgeage en ligne [12].

Fig. 14. Crater end forging.

\subsubsection{Les raisons métallurgiques de l'introduction de la} réduction en ligne

La réduction en ligne apparaît tardivement sur les machines de coulée en réponse aux problèmes posés par le passage en $\mathrm{CC}$ des nuances à tendances fortes à la ségrégation centrale, notamment sur les produits de forte épaisseur. Elle résulte des exigences croissantes des mécaniciens à propos de la tenue en service du matériau acier mis en oeuvre et notamment la recherche de l'amélioration de l'homogénéité et de l'isotropie des propriétés. Les critères de la mécanique de la rupture ou ceux de l'endurance en fatigue à des matériaux issus de la coulée continue sont d'autant plus sensibles à la présence de ségrégation majeure centrale qu'il s'agit le plus souvent de nuances à hautes caractéristiques mécaniques (pour lesquelles la taille des défauts critiques diminue avec l'élévation des caractéristiques) et aussi souvent à fortes teneurs en carbone. Les progrès considérables en matière de propreté inclusionnaire ont fini par mettre en évidence, sur les propriétés d'emploi (notamment en matière d'endurance) les effets nocifs des hétérogénéités compositionnelles et structurales résiduelles au cœur des produits coulés (ségrégations, carbures...) et ont contraint les aciéristes à les traiter sérieusement.
Deux produits sidérurgiques sont à cet égard emblématiques de ces exigences : le fil steelcord (nuances d'aciers durs à teneurs en carbone comprises entre $0,7 \%$ et $1 \%$ ) utilisé en renfort de pneumatiques et les nuances pour roulements (fils pour corps roulants et barres ou tubes pour bagues). L'augmentation considérable des exigences en matière de durée de vie des équipements (pneumatiques pour poids lourds, roulements...) ont conduit à évaluer le comportement de ces matériaux dans le domaine de la fatigue gigacyclique $\left(10^{9}\right.$ cycles $)$ et fait apparaître de nouveaux mécanismes d'endommagement.

Le même type d'exigences s'est posé pour les très gros produits destinés à la construction mécanique lourde (tôles fortes pour la chaudronnerie lourde, gros ronds à tubes utilisés notamment dans l'exploitation pétrolière...) avec la circonstance aggravante que la ségrégation centrale majeure croissait avec la taille du produit coulé. Ce sont ces problèmes qui ont conduit à la mise au point de la réduction mécanique forte (MHR) sur les machines coulant des produits de forte épaisseur.

\subsection{La coulée des produits de forte section}

Elle concerne la production des tôles fortes mais aussi les produits longs de forte section (gros blooms et gros ronds). 
Tableau 3. Caractéristiques de quelques machines à gros produits d'après [12].

Table 3. Characteristics of a few big products machines.

\begin{tabular}{llll}
\hline Société/ usine & Type/nb. lignes & Format $(\mathrm{mm})$ & Contrôle de la ségrégation \\
\hline Sanyo/Himeji & $\mathrm{V} / 3$ & $370 \times 470$ & SEMS/ MSR \\
Daïdo/Chita & $\mathrm{V} / 4$ & $\varphi 350$ & SEMS/ MSR \\
Kobe/Kobe & $\mathrm{VC} / 2$ & $300 \times 430$ & SEMS/ MHR \\
Sumitomo/Wakayama & $\mathrm{C} / 4$ & $415 \times 530$ & SEMS/ MSR \\
NKK/Keihin & $\mathrm{C} / 6$ & $\varphi 325$ & SEMS/ MSR \\
Timken/Faircrest & $\mathrm{V} / 3$ & $460 \times 610$ & SEMS/ DMSR \\
Kawasaki/Mizushima & $\mathrm{C} / 4$ & $400 \times 560$ & SEMS/ MHR \\
Dilling (CC5) & $\mathrm{V} \mathrm{C} / 2$ & $450 \times 2200$ & SEMS/ DMSR \\
Dilling (CC6) & $\mathrm{V} \mathrm{C} / 2$ & $500 \times 2200$ & SEMS/ DMSR \\
\hline
\end{tabular}

$\mathrm{V}$ : machine verticale; $\mathrm{C}$ : machine courbe; $\mathrm{VC}$ : machine verticale/courbe.

Ces produits sont le plus souvent destinés à la construction mécanique lourde (plateformes pétrolières offshore, tabliers de ponts, installations sous pression, masses-tiges de forage, construction navale...) qui impose en général des exigences sévères en matière d'homogénéité et d'isotropie des propriétés mécaniques.

Ce secteur de la production sidérurgique s'est intéressé très tôt à la coulée continue, la taille des produits coulables croissant avec les perfectionnements apportés aux machines. Ceci explique que la croissance des épaisseurs coulées se soit faite pas à pas, à l'occasion des reconstructions de machines. En effet la lourdeur et la complexité des machines à concevoir ainsi que l'ampleur des problèmes de ségrégation à maîtriser, croissait avec la taille des produits coulés (d'autant qu'il s'agissait le plus souvent de couler des nuances à haut ou moyen carbone particulièrement affectées par la ségrégation majeure). La problématique était double:

- machine droite/machine courbe? La machine droite va conduire à une très grande hauteur. La machine courbe va poser la question du cintrage/décintrage et des efforts importants à exercer compte tenu de la taille des produits à déformer. La propreté inclusionnaire peut être affectée par une courbure trop précoce de la ligne (cf. Partie II). Le compromis sera souvent trouvé (pour les brames notamment) avec la machine verticale/courbe présentant une partie verticale de longueur suffisante sous la lingotière;

- l'application de la MHR va imposer des efforts de déformation proches de ceux d'un forgeage à chaud, puisqu'il est nécessaire que l'effet de compression se manifeste jusqu'à cœur du produit. Il en résulte que les outils chargés d'appliquer une telle déformation devront avoir une forte puissance, qu'ils alourdiront considérablement la machine de coulée et qu'ils introduiront une modification sensible de la géométrie du produit avec éventuellement des risques sérieux d'endommagement si la MHR est appliquée sans discernement.

Concevoir et exploiter de telles machines revient donc à associer les savoir-faire du forgeron et du spécialiste de coulée continue et à les rendre compatibles sur un même équipement ce qui n'est pas des plus simples. Les constructeurs tels que SMS Concast A.G. et certains Japonais ont maîtrisé ces problèmes en associant avec précision brassage électromagnétique secondaire et compression mécanique, le tout piloté à partir d'une modélisation de la solidification (DMSR : Dynamic Mechanic Soft Reduction). Le tableau 3 donne, à titre d'exemples, les caractéristiques de quelques machines destinées à la coulée de gros produits, appliquant diverses techniques de réduction en ligne.

La figure 15 schématise une des machines verticale/ courbe de Dilling destinée à la coulée de brames jusqu'à $500 \mathrm{~mm}$ d'épaisseur.

On peut cependant s'interroger sur l'épaisseur de produit au-delà de laquelle l'alourdissement et la complexification de la machine de coulée continue, par l'introduction de la MHR, rendent le maintien d'un blooming plus pertinent, y compris en conservant une voie lingot. C'est ainsi que l'usine Industeel de Châteauneuf (Loire) (Industeel appartenant au groupe Arcelor-Mittal) livrant des plaques hyperlourdes, reste en voie lingots, ceux-ci étant éventuellement dégrossis à la presse à forger avant laminage. De même Dilling (le plus gros producteur européen de tôles fortes) continue à couler en lingots (jusqu'à $60 \mathrm{t}$ ) le métal destiné à ses plus gros produits, malgré l'existence de ses machines à brames de forte épaisseur.

\subsection{Conclusion partielle sur la solidification en machine}

À l'issue de cet examen de la solidification en machine de coulée, on constate que l'aciériste ne dispose fondamentalement que de trois paramètres principaux pour optimiser les structures de solidification : la surchauffe, la vitesse de coulée (c. à d. la vitesse d'extraction du produit) et le taux de refroidissement secondaire que l'on exprime traditionnellement en volume d'eau pulvérisé rapporté à la masse de métal coulé: litre $/ \mathrm{kg}$. Nous savons, d'une part, que le contrôle de ces paramètres ne suffira jamais à totalement effacer les hétérogénéités compositionnelles et structurales engendrées par le processus de solidification en machine et, d'autre part, que l'exploitant aura tendance à couler, pour des raisons de productivité, à la plus grande vitesse permise 


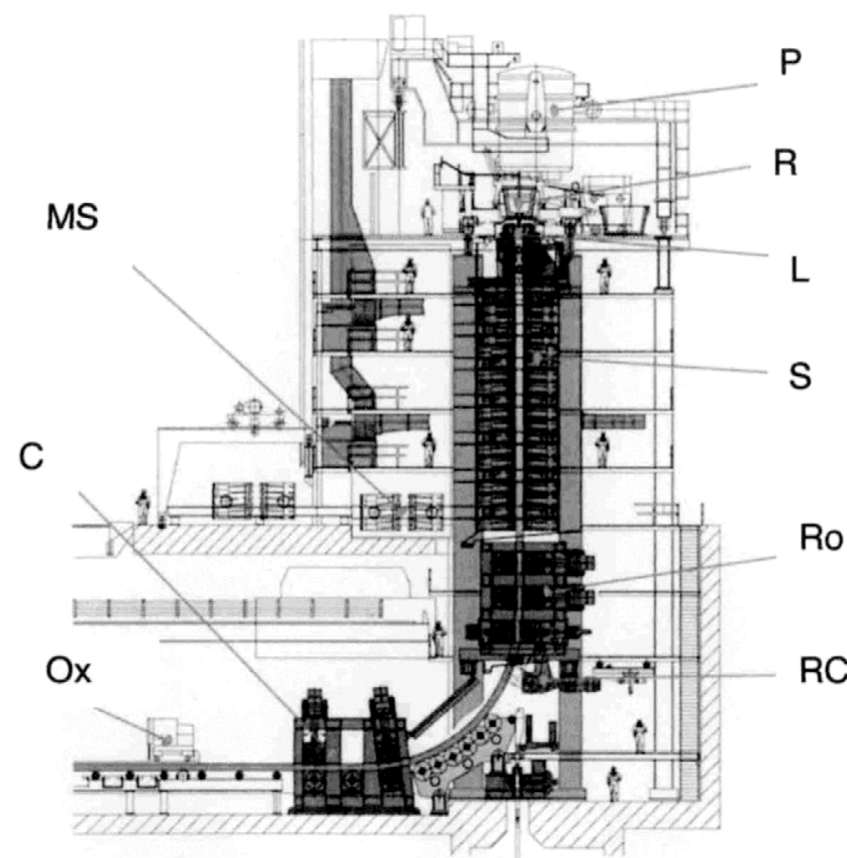

Fig. 15. Machine verticale courbe pour brames de forte épaisseur [13]. $\mathrm{P}$ : poche; $\mathrm{R}$ : répartiteur; $\mathrm{L}$ : lingotière; $\mathrm{S}$ : segments de guidage et refroidissement secondaire; Ro: rouleaux de réduction; RC : rouleau de cintrage; MS manipulateur des segments ; $\mathrm{C}$ unité de décintrage et d'extraction; Ox: installation d'oxycoupage.

Fig. 15. Vertical/Curved casting machine for heavy slabs.

par l'équipement et que tous les désordres sur la machine finiront par produire des défauts de solidification ayant des conséquences plus ou moins nocives sur le produit final. Selon les exigences qualitatives posées par les nuances d'aciers coulées, l'aciériste aura donc recours à des moyens additionnels qui devront être prévus dès le design de la machine: brassages électromagnétiques primaire et secondaire, réduction en ligne... On comprend dès lors pourquoi il y a une considérable différence de complexité entre une machine coulant des aciers pour roulements et celles destinées aux ronds à béton. Chaque aciérie est un cas particulier nécessitant une adaptation du type de machine au carnet des nuances produites et des demi-produits livrés.

Dans les paragraphes suivants, nous examinerons quelques-uns de ces problèmes spécifiques posés par quelques familles de nuances particulières dont le choix nous a été dicté soit par leur grande diffusion, soit par leur comportement particulier à la solidification.

\section{La coulée continue des aciers effervescents}

C'est une famille de nuances qui a disparu des catalogues des sidérurgistes depuis plus d'un demi-siècle. Au début de la phase d'industrialisation du procédé de coulée continue, ce type d'acier occupait encore une place suffisamment importante, pour que les aciéristes tentent de le couler en continu. À ce titre, le sujet mérite d'être brièvement évoqué.

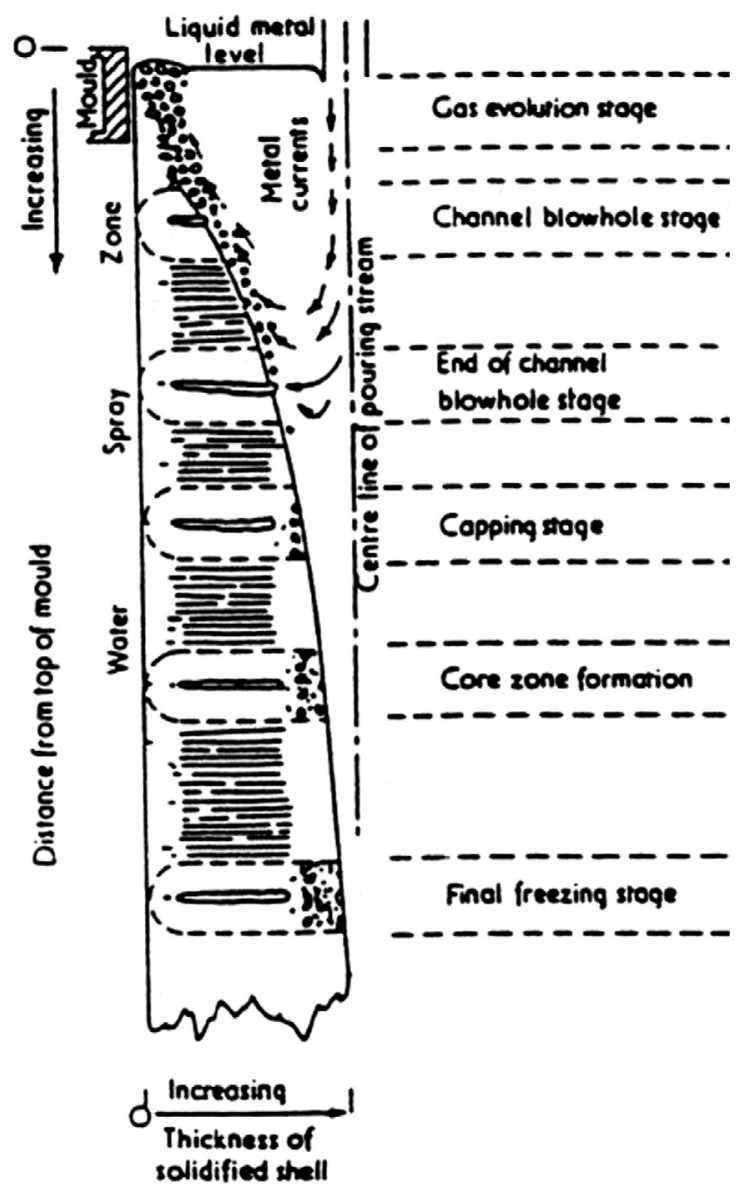

Fig. 16. Modèle de solidification d'un acier effervescent en coulée continue [14-17].

Fig. 16. Rimmed steel solidification in continuous casting.

Rappelons que l'acier effervescent est peu désoxydé lors de son élaboration pour que son contenu en oxygène dissous soit suffisamment élevé et qu'à la solidification on observe une reprise de la réaction du carbone sur l'oxygène dissous avec émission de CO (d'où le nom d'acier effervescent). À une époque où l'aciériste était particulièrement démuni en matière de maîtrise de la propreté inclusionnaire, une telle pratique permettait de renoncer en partie à la désoxydation par précipitation et d'évacuer ainsi une bonne partie des problèmes posés par les inclusions non métalliques. La contrepartie était l'obtention d'un lingot truffé de soufflures (de $\mathrm{CO}$ ), mais qui se refermaient sans problèmes lors du laminage (puisque leurs surfaces étaient non oxydées). La peau et le «lard » de ces lingots présentaient une qualité exceptionnelle au point d'en faire alors un produit de choix pour les aciers plats destinés à l'emboutissage.

Comment allait se comporter un tel acier effervescent sur une ligne de CC et notamment en lingotière? C'est la société Inland Steel aux USA qui en 1956 réussit à définir les conditions précises d'équilibre à réaliser entre l'activité de l'oxygène dans l'acier et le taux de refroidissement appliqué de manière à répartir convenablement les soufflures dans le produit coulé (Fig. 16). Cependant, la coulée continue de ce type de nuances resta suffisamment 
problématique pour que le complexe sidérurgique SOLMER (aujourd'hui ARCELOR-MITTAL) qui sera construit à Fos/Mer au début des années 1970 repose initialement sur la coulée en lingots (et donc requière la présence d'un laminoir blooming). La mise au point de l'élaboration de nuances à très basse teneur en carbone, traitées sous vide et désoxydées à l'aluminium permettra de retrouver puis de dépasser largement la qualité des nuances effervescentes pour produits plats minces emboutissables et ouvrira la voie à la coulée continue, Solmer s'équipera de sa première machine de CC en 1975 d'abord pour couler des nuances courantes.

\section{La coulée continue des aciers à bas carbone pour produits plats minces destinés au laminage à froid}

Ces aciers représentent une proportion très importante de la production sidérurgique et concernent notamment les produits emboutissables pour l'automobile et les aciers pour emballage, dont les boîtes de boissons.

\subsection{Exigences qualitatives spécifiques}

Ces aciers sont coulés sur machines à brames. Les brames sont ensuite laminées à chaud sur un train à bande (TAB) pour produire des bobines de tôle dont l'épaisseur peut descendre jusqu'à 1,2 mm. Les bobines à chaud sont reprises en laminage à froid pour descendre jusqu'à des épaisseurs de $0,15 \mathrm{~mm}$. Le laminage à froid est le plus souvent suivi d'un revêtement de surface souvent complexe par immersion à chaud, électrodéposition, enduction (galvanisation, étamage, chromage, prélaquage...). Le traitement de ces aciers à l'usine de laminage à froid (y compris les revêtements de surface), et leur mise en œuvre par déformation à froid (emboutissage et découpage) imposent des exigences sévères, notamment en matière d'état de surface et de propreté inclusionnaire. Ce sont sur les machines à brames coulant de tels aciers qu'ont été réalisés les principaux progrès en matière de qualité de surface (coulée sous laitier et cycle d'oscillation...), grâce à la maîtrise du fonctionnement de la tête de machine, ainsi que les principaux progrès en matière de productivité: vitesse de coulée, longueur des séquences, changement de format en cours de coulée. Les développements en matière de maîtrise des pratiques en lingotière pour assurer un bon état de surface des brames ont été décrits en partie II.

En ce qui concerne la propreté inclusionnaire en sidérurgie, rappelons trois points concernant particulièrement les produits plats minces [1]:

\footnotetext{
- la lutte contre un contenu inclusionnaire excessif doit être une préoccupation permanente non seulement pour éviter les contaminations tardives (réoxydations, entrâิnements du laitier de coulée), mais encore pour profiter de toutes les étapes du processus pour piéger les inclusions résiduelles (en particulier lors des circulations du métal en répartiteur et en lingotière). Cette démarche
}

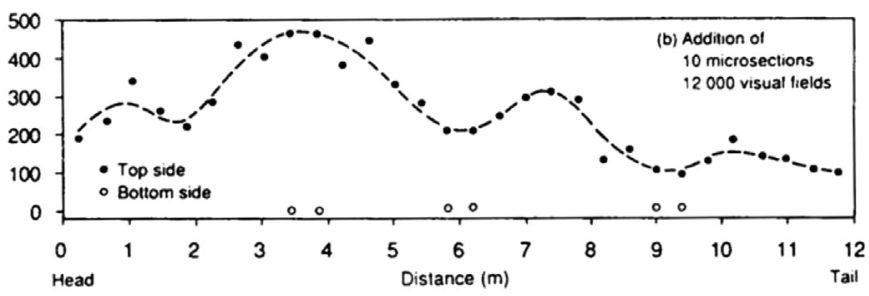

Fig. 17. Propreté sur une brame de $12 \mathrm{~m}$ de long [18]. Nombre de clusters d'alumine en fonction de la distance.

Fig. 17. Cleanliness along a $12 \mathrm{~m}$ long slab.

vers la haute propreté inclusionnaire fut grandement facilitée par les développements de la métallurgie secondaire en poche, entre le réacteur primaire (convertisseur ou four électrique) et la coulée, avec notamment les traitements en poche sous vide;

- le problème longtemps posé par les machines courbes (lorsqu'on coulait un métal à la propreté mal maîtrisée) dans lesquelles les inclusions décantaient au plafond de la brame (Fig. 17) au lieu d'atteindre le ménisque (dans une machine droite) a été réglé successivement par des machines verticales/courbes, puis à lingotière courbe à rayon suffisamment grand, mais surtout par des progrès très significatifs au niveau de l'élaboration: désoxydation par le carbone sous vide dans un dégazeur par circulation type $\mathrm{RH}$, ce qui permettait de réduire fortement la désoxydation par précipitation;

- le problème du colmatage des busettes de coulée par des dépôts d'inclusions d'alumine dans les aciers calmés à l'aluminium. Ces dépôts avaient deux conséquences néfastes : ils réduisaient progressivement le débit d'acier coulé avec impossibilité de respecter la consigne de vitesse de coulée et éventuellement, dans les cas extrêmes, perte d'une ligne de coulée et même impossibilité d'achever la coulée de la poche d'acier. En outre, ces amas d'alumine déposés dans les busettes se détachaient de manière aléatoire, étaient entraînés dans le flux d'acier pour donner des amas inclusionnaires de grande taille dans le produit solidifié. Le problème sera réglé, pendant le traitement de l'acier en poche, par des injections d'alliage SiCa qui transforment les inclusions d'alumine en aluminate de chaux $\left(\mathrm{Al}_{2} \mathrm{O}_{3}, \mathrm{CaO}\right)$ ne se déposant pas dans les busettes.

\subsection{La solidification péritectique des aciers bas carbone, ses conséquences}

Les aciers que nous considérons dans ce paragraphe sont à bas carbone $(<0,2 \%)$. La solidification de ces aciers (dits péritectiques) a des conséquences suffisamment importantes en coulée continue pour que nous l'évoquions avec précision. En effet, en plus de la contraction liquide-solide, la transformation $\delta-\gamma$ produit une nouvelle contraction et une baisse de la conductibilité thermique de la peau solidifiée. Les aciers à très bas carbone (dits ULC, jusqu'à 


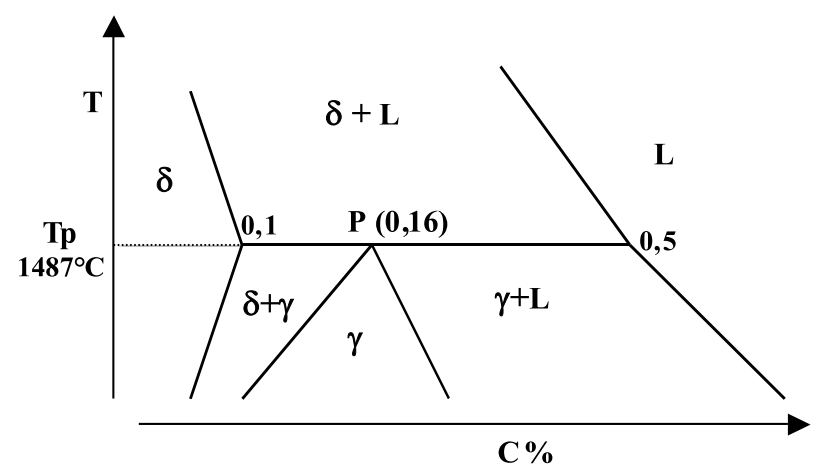

Fig. 18. Schéma du diagramme de phase Fe-C au voisinage de la réaction péritectique.

Fig. 18. Fe-C phase diagram near the peritectic reaction.

0,003\% C) ne passent pas par la réaction péritectique, mais encaissent complètement la transformation $\delta-\gamma$.

\subsubsection{Solidification dans le diagramme fer-carbone aux très basses teneurs en carbone}

Dans le domaine de teneurs en carbone de 0,1 à $0,5 \% \mathrm{C}$, la solidification met en jeu le palier péritectique à $1487^{\circ} \mathrm{C}$ (température du diagramme d'équilibre Fe-C) (Fig. 18). La complexité de cette réaction vient du fait que de part et d'autre de la teneur en carbone de $0,16 \% \mathrm{C}$ (variable selon les autres éléments présents), la succession des réactions est très différente. Dans tous les cas, la solidification commence en $\delta$ et progresse jusqu'à la température péritectique Tp. À ce moment, les chemins divergent selon que la teneur en $\mathrm{C}$ est inférieure ou supérieure à $\mathrm{P}$ :

- en dessous de $0,1 \% \mathrm{C}$, la réaction péritectique n'est pas active, la solidification se produit complètement en phase $\delta$ et la transformation en $\gamma$ ne met en jeu que des solides à des températures plus basses ;

- entre 0,1 et 0,16\% C, la solidification est terminée à Tp. Ensuite, se déroule la transformation de $\delta$ en $\gamma$ qui se traduit par une contraction (de cubique centré en cubique face centrée). Plus la teneur en carbone est faible, plus la proportion de $\delta$ solidifié est importante, avant la réaction péritectique à $\mathrm{Tp}$.

Ensuite, la transformation $\delta-\gamma$ se produit progressivement pendant le refroidissement :

- à $0,16 \% \mathrm{C}$, la fin de solidification et la transformation totale de $\delta$ en $\gamma$ se produisent simultanément selon la réaction péritectique:

$(85 \%) \delta(0,1 \% \mathrm{C})+(15 \%$ liquide $)(0,5 \% \mathrm{C})=>(100 \%)$ $\gamma(0,16 \% \mathrm{C})$;

- au-dessus de $0,16 \% \mathrm{C}$, la solidification continue après la réaction péritectique à Tp qui voit la disparition de $\delta$ solide, servant de germe à $\gamma$. La solidification se poursuit dans le domaine $\gamma+$ liquide ;

- au-delà de $0,5 \% \mathrm{C}$, la solidification se produit en $\gamma$, de façon habituelle.

Cette description à partir du diagramme de phases d'équilibre ne correspond pas tout à fait aux conditions de solidification des aciers industriels, pour lesquels la réaction

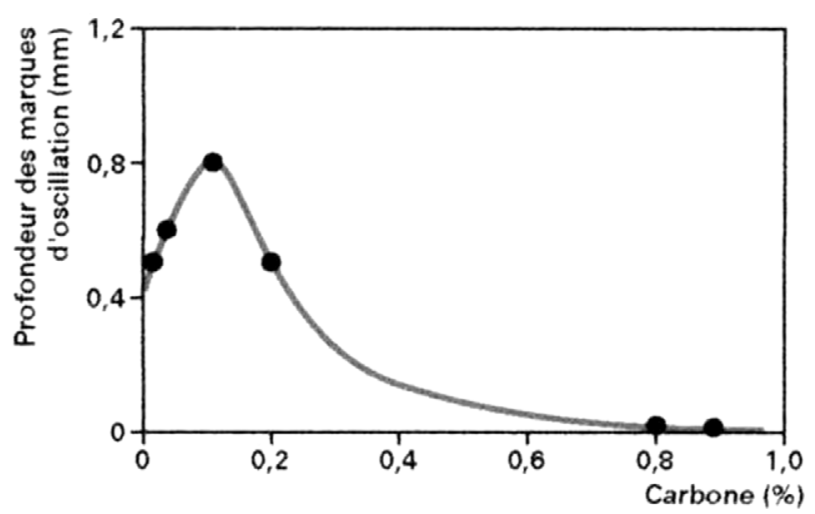

Fig. 19. Profondeur des marques d'oscillation en fonction de la teneur en carbone [4].

Fig. 19. Depth of oscillation marks as a function of Carbon content.

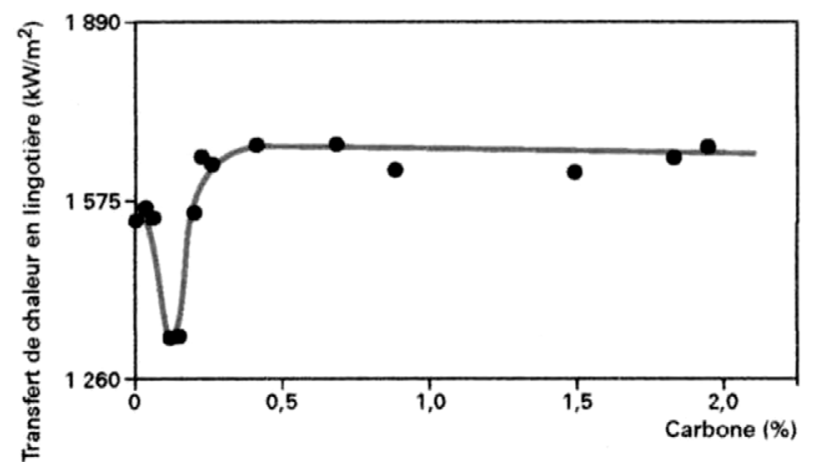

Fig. 20. Transfert de chaleur en lingotière en fonction de la teneur en carbone [4].

Fig. 20. Heat transfer in the mould as a function of the carbon content.

péritectique est déplacée en fonction de la vitesse de solidification, mais les principes généraux restent les mêmes.

\subsubsection{Conséquences sur la qualité de surface des brames}

Rides d'oscillation et décollement en lingotière: la contraction qui accompagne la transformation $\delta-\gamma$ dans la peau solidifiée (en plus de la contraction liquide/solide) entraîne des décollements entre le métal et la lingotière, qui produisent des rides superficielles à l'origine de défauts. Ce phénomène est particulièrement important pour les aciers à $0,1 \%-0,16 \%$ C , c'est-à-dire à gauche de la composition péritectique, dans le domaine de composition où la solidification en $\delta$ se termine à Tp avec, simultanément la transformation $\delta-\gamma$ (Fig. 19).

De plus, le flux thermique extrait par la lingotière refroidie subit une diminution importante dans ce même domaine de composition (Fig. 20). C'est le résultat du décollement en lingotière par la contraction de $\delta$ en $\gamma$, mais aussi de la plus faible conductibilité thermique de $\gamma$ par rapport à $\delta$. 
Les figures 19 et 20 montrent l'effet de la teneur en carbone sur les défauts de surface et sur le transfert de chaleur à la lingotière. Le maximum de problèmes se situe au point péritectique $(0,16 \% \mathrm{C})$, mais on constate que les aciers à très bas carbone sont également affectés, par comparaison avec les nuances à plus de $0,2 \% \mathrm{C}$.

\subsubsection{Fissuration des aciers bas $C$ à la coulée}

L'apparition de fissures longitudinales et de marques d'oscillation très prononcées est particulièrement critique pour les aciers de teneurs en carbone inférieures à $0,20 \% \mathrm{C}$, particulièrement entre 0,12 et $0,16 \% \mathrm{C}$ où l'effet passe par un maximum. Ces problèmes sont liés à la faible résistance en traction de la ferrite delta à haute température qui forme la première peau pendant la solidification en lingotière. Les propriétés mécaniques à chaud de la ferrite delta sont basses, en comparaison de celles de l'austénite. Une étude faite à $1450^{\circ} \mathrm{C}$, citée par Wolf [19], donne $5 \mathrm{MPa}$ pour un acier à très bas carbone $(0,03 \% \mathrm{C})$, alors qu'elle est 4 fois plus élevée pour l'austénite : $20 \mathrm{MPa}$ dans un acier à $0,10 \%$ C. Ces valeurs, qui concernent le métal solide à $1450^{\circ} \mathrm{C}$, peuvent s'extrapoler vers les températures plus élevées voisines du liquidus où se forme la première peau en lingotière, et où la résistance mécanique de la ferrite delta est encore plus faible. La synthèse critique de Wolf aboutit à des recommandations sur le contrôle de la stabilité du ménisque qui conditionne la qualité de surface des aciers à bas carbone.

\section{La coulée continue des aciers inoxydables}

\subsection{Problèmes spécifiques posés par la coulée continue des aciers inoxydables}

La coulée continue des aciers inoxydables, tout particulièrement en produits plats minces (nuances ferritiques type AISI 430, nuances austénitiques type AISI 304 ou AISI 316 ou stabilisées Ti comme la nuance AISI 321), posa quelques problèmes spécifiques pour au moins deux raisons :

- les exigences de qualité de surface du produit fini en acier inoxydable sont particulièrement sévères. Il importait donc que la qualité de surface du produit brut de coulée en sortie machine soit particulièrement bonne, non seulement pour ne pas engendrer de défauts lors du laminage à chaud au TAB ou lors du laminage à froid ultérieur, mais encore pour que ces défauts résiduels ne soient pas des sites de corrosion préférentielle sur le produit mis en œuvre;

- les réparations des défauts de surface sur produits inoxydables bruts de coulée ne peuvent se faire que par meulage (et non par scarfing au chalumeau comme sur les produits non inoxydables). C'est une opération particulièrement lourde et onéreuse si les surfaces à meuler sont importantes (la coulée continue des produits plats en aciers inoxydables a commencé avec un meulage des brames coulées, proche de $100 \%$, opération dite d'épaillage).

La maîtrise de la qualité de surface était d'autant plus délicate que les aciers inoxydables présentaient des caractéristiques métallurgiques suffisamment spécifiques pour nécessiter une adaptation sinon des technologies, au moins des pratiques mises en œuvre sur une machine de coulée destinée aux aciers faiblement alliés.

\subsection{Solidification des aciers inoxydables}

Dans les nuances ferritiques (type AISI 430), la solidification directement en ferrite et la conservation de cette structure au cours du refroidissement favorisent la ségrégation du carbone (peu soluble dans la ferrite) d'où l'apparition de filets ségrégés et éventuellement de criques. La résistance mécanique à chaud de la structure ferritique est faible (divisée par un facteur 5 par rapport à une structure austénitique à la même température), ce qui peut conduire à la formation de criques consécutives aux contraintes diverses engendrées sur le produit par la machine de coulée. Cette double spécificité a fait considérer par certains industriels, jusqu'au début des années 1970, que les nuances ferritiques (type AISI 430) n'étaient pas coulables dans de bonnes conditions en coulée continue.

Les nuances austénitiques (type $18 / 8$ et $18 / 8$ Mo: AISI $304,316 \ldots)$ ont une solidification commençante en ferrite $(\delta)$ ou biphasée $(\delta+\gamma)$ (sauf celles qui sont les plus riches en $\mathrm{Ni})$; avec transformation en austénite quelques dizaines de degré sous le solidus [20]. Il en résulte une structure de la matrice brute de coulée très partiellement biphasée avec des îlots de ferrite $\delta$ ayant tendance, selon leur composition (nuances contenant du Mo en particulier), à se transformer à plus basse température en phase $\sigma$ pouvant engendrer une fragilité spécifique à chaud. Cependant, on équilibre la structure austénitique avec quelques $\%$ de ferrite $\delta$ pour éviter les décohésions intergranulaires au décintrage et à la mise en forme à chaud.

Comme pour la solidification péritectique des aciers à bas carbone, la transformation $\delta / \gamma$ produit une contraction qui peut se traduire par des décollements de la peau solidifiée contre la lingotière et produire des rides superficielles. Par ailleurs la conductivité thermique de l'austénite fortement alliée (aciers inoxydables austénitiques) est notablement diminuée par rapport à celle de la ferrite et de l'austénite non alliées (aciers au carbone); ce qui peut conduire à une adaptation des vitesses de coulée et des politiques de refroidissement sur les nuances austénitiques, cependant dans le 304 on observe peu de criques axiales en raison d'une bonne ductilité et d'une résistance élevée [21]. La structure finale d'un acier 304 est illustrée dans la figure 21 sur une billette de coulée continue rotative. L'image de droite montre la structure de solidification après une attaque Fry mettant en évidence les deux zones basaltique et équiaxe. L'image de gauche, après attaque acide, montre la structure de recristallisation, après la transformation de $\delta$ en $\gamma$, qui n'est pas celle des dendrites primaires [22], contrairement aux apparences.

Les austénites peuvent présenter, du fait des impuretés résiduelles ( $\mathrm{S}, \mathrm{Sn}, \mathrm{Bi}$, apportées par les ferrailles refondues) ségrégées aux joints de grains, des ductilités déficientes aux températures moyennes $\left(1000^{\circ} \mathrm{C}-1200{ }^{\circ} \mathrm{C}\right)$, ce qui peut poser des problèmes au décintrage. 


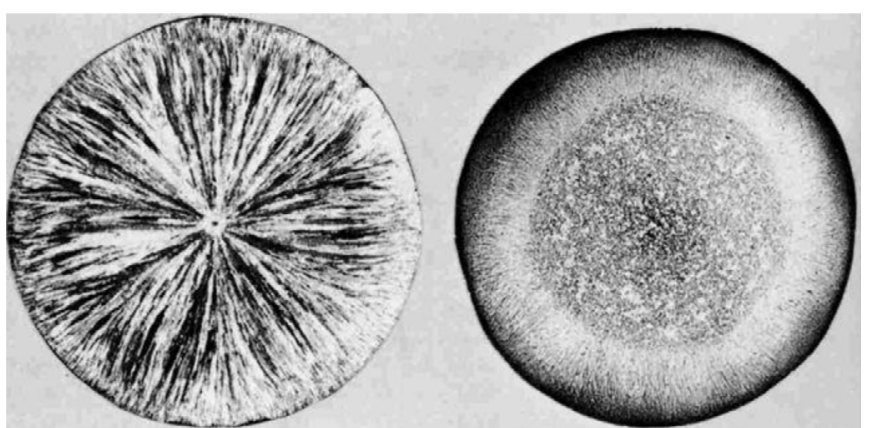

Fig. 21. Structures de solidification d'un acier inoxydable austénitique en coulée continue rotative [22].

Fig. 21. Solidification structure of austenitic stainless steel in rotating continuous casting.

On notera à propos des produits longs en aciers inoxydables que les opérations de l'industrie pétrolière en offshore profond font appel, pour des raisons de mise en œuvre du matériau dans des milieux extrêmement corrosifs (température, pression, acidité...), à des tubes en nuances inoxydables super-austénitiques (type 25/20 saturées en azote pour relever les caractéristiques mécaniques) ou austéno-ferritiques (nuances dites duplex). Ces nuances concentrent les difficultés que nous avons énoncées précédemment, elles sont cependant coulées en continu sur machines verticales à blooms (machine CC d'UGITECH).

Les problèmes que nous venons d'évoquer ont été progressivement maîtrisés à partir du milieu des années 1970. On notera que la période de développement de la CC pour les aciers inoxydables a coïncidé avec celle de la généralisation du procédé d'affinage AOD [23] qui en améliorant grandement la qualité du métal coulé (désulfuration, maîtrise de l'état inclusionnaire) a facilité le passage en coulée continue.

\subsection{Aciers inoxydables et coulée continue, évolution de la situation française}

Il n'est pas sans intérêt, d'un point de vue technicoéconomique, d'examiner la pénétration en France de la coulée continue dans les filières de production des aciers inoxydables, car elle est très révélatrice des bouleversements que peuvent induire des percées technologiques dans un contexte fortement concurrentiel, lié aux premières manifestations de la mondialisation. Le contenu de ce paragraphe est à mettre en relation avec l'article publié dans la revue Matériaux et Techniques, traitant de l'histoire de la fusion électrique [23]. Un tel examen illustrera le fait que les efforts de développement technique ne doivent pas cesser, et de facto ne cessent pas, même dans un contexte de forte restructuration au bénéfice des sites les mieux équipés.

Le point de départ de l'histoire que nous voulons retracer se situe dans la seconde partie des années 1960. Le leader technico-économique européen en matière de production d'aciers inoxydables est alors la société Ugine avec trois usines : l'aciérie d'Ugine (73) spécialisées sur les produits longs (barres et fils), l'aciérie de Moutiers (73) (aujourd'hui démantelée) coulant des lingots destinés aux produits longs et aux produits plats, l'aciérie de l'Ardoise (30) (aujourd'hui démantelée) coulant des lingots destinés aux produits plats. La suprématie technique de la société Ugine tient alors à la maîtrise de deux procédés: la production du ferrochrome suraffiné ( $\mathrm{FeCr}$ à très bas carbone) par silicothermie couplée à une carbothermie selon le procédé Perrin-Greffe [23] et la coulée sous laitier des lingots qui garantit à ceux-ci une haute qualité de surface. Ces deux procédés sont le reflet d'une maîtrise exceptionnelle pour l'époque, des échanges métal/laitier. Les deux usines de l'Ardoise et de Moutiers comportaient en amont de l'aciérie électrique des unités de production de $\mathrm{FeCr}$ suraffiné qui permettaient d'alimenter les fours de l'aciérie en $\mathrm{FeCr}$ liquide à très basse teneur en carbone. Les lingots méplats coulés dans ces aciéries et destinés aux produits plats étaient transformés en brames sur le train slabbing de Sollac Fos, ces brames étaient ensuite laminées sur le train continu à chaud pour donner les coils qui étaient laminés à froid dans les ateliers de Gueugnon (71). Cette situation était d'autant plus confortable pour Ugine que ses principaux concurrents en matière d'aciers inoxydables étaient tributaires des producteurs de FeCr suraffiné solide, particulièrement de la société Ugine qui mettait une partie de sa production de ferro-alliages des usines de Moutiers et l'Ardoise sur le marché. La maîtrise technique d'Ugine sur toute la chaîne de production depuis les matières premières, y compris le minerai de $\mathrm{Cr}$, en faisait, à la fin des années 1960, le premier producteur mondial d'aciers inoxydables. Ses principaux concurrents sur le marché européen étaient des producteurs allemands, britanniques, scandinaves; sur le marché mondial les USA et le Japon et sur le marché français, en produits plats, la société Chatillon-Commentry-Biache (CCB) par son usine d'Isbergues (qui produisait aussi des aciers au $\mathrm{Si}$ à grains orientés pour l'industrie électrotechnique). Le marché des aciers inoxydables (notamment en produits plats) était alors en très forte croissance pour couvrir les besoins du bâtiment (décoration, couvertures...), de l'industrie chimique (cuves de réacteurs, réservoirs de stockage, canalisations), de l'industrie agro-alimentaire, de l'électroménager...

Cette situation va être radicalement bouleversée par l'apparition de l'affinage AOD qui permet de se passer, du FeCr suraffiné (bas carbone) et ouvre la production des aciers inoxydables à tout aciériste disposant d'un four électrique de fusion, s'équipant d'une cornue AOD et d'une coulée continue, dont on a vu, au début de cet article, les avantages concurrentiels. Cette double révolution technologique (l'affinage AOD et la coulée continue) rend donc rapidement obsolètes les deux procédés qui avaient conduit à la suprématie d'Ugine en matière d'élaboration des aciers inoxydables.

La société Chatillon-Commentry-Biache (CCB), calquant son développement sur celui de Jones and Laughlin aux USA, implante à Isbergues une coulée continue de brames dès 1972. L'implantation du réacteur d'affinage AOD suit en 1975. En 1974, CCB a pris une participation dans le train à bandes en construction à Charleroi (Carlam). La France dispose donc de deux filières concurrentes en produits plats inox: l'une opérée par 
Ugine (selon la filière lingots) faisant effectuer ses laminages à chaud chez Sollac Fos (qui dispose d'un blooming), l'autre par CCB (selon la filière CC) faisant laminer en Belgique chez Carlam. De très nombreuses usines de par le monde vont adopter ce schéma de production basé sur le réacteur AOD et la coulée continue, banalisant ainsi la production des aciers inoxydables. Il faudra cependant attendre 1983, soit dix ans après Isbergues pour que l'usine de l'Ardoise s'équipe d'une coulée continue. Entre temps, Ugine avait regardé d'assez près la faisabilité de la coulée de brames minces selon le procédé Hazelett (partie IV), sans l'adopter [27].

Les restructurations consécutives à la création d'Arcelor en 2002 conduiront au regroupement de la production des produits plats inoxydables sur le site de Charleroi avec l'arrêt des sites de L'Ardoise puis d'Isbergues; mais fin 1989, sous l'impulsion de F. Mer Usinor-UgineSA lancera sur le site d'Isbergues la construction du prototype industriel Myosotis de coulée de bandes (démarrage en 1991) qui prendra la suite des pilotes ayant fonctionné à l'Irsid (partie IV). En produit longs inoxydables, le passage en coulée continue se fera en 1978 à Imphy (aujourd'hui APERAM) avec une machine rotative et à Ugine Savoie (aujourd'hui UGITECH) en 1982 avec une machine à blooms, ces deux machines sont verticales.

\section{La coulée continue des aciers à roulements}

\subsection{La spécificité des aciers à roulement vis-à-vis de la CC}

Nous choisissons cette famille d'aciers pour illustrer les problèmes que pose la coulée continue des nuances à haute teneur en carbone, à grand intervalle de solidification et donc à forte tendance à la ségrégation.

La nuance 100Cr6 est une des deux principales nuances (l'autre étant la nuance de cémentation $20 \mathrm{Ni}$ Cr Mo 2) mises en œuvre dans la fabrication des roulements que ce soit pour les corps roulants à partir de fils, ou pour les bagues forgées à partir de lopins prélevés dans des barres laminées ou dans des tubes sans soudure. Les fabricants de roulements s'opposèrent longtemps à ce que cette nuance soit produite par la filière coulée continue. Cette opposition à la coulée continue de la clientèle des fabricants de roulements était si déterminée et paraissait si métallurgiquement fondée que la société UGINE (qui produisait alors $80 \%$ des aciers à roulements consommés en France et livrait ces aciers en Europe et aux USA), lors de la construction de sa nouvelle aciérie électrique à Fos/Mer au début des années 1970 misait sur la conservation d'un bassin de coulée en lingots. La raison métallurgique qui dictait ce choix était double:

- la première tenait aux exigences de propreté inclusionnaire, particulièrement sévères, induites par les performances en endurance exigées des roulements et que la coulée continue avait alors beaucoup de mal à satisfaire [1] ; - la seconde résidait dans l'importance des ségrégations à cœur des produits solidifiés en continu liées à la présence de $\mathrm{Cr}(1,5 \%)$ et à la forte teneur en $\mathrm{C}(1 \%)$ de la nuance, ce qui entraînait, par l'effet de la ségrégation majeure, la formation en fin de solidification de carbures primaires

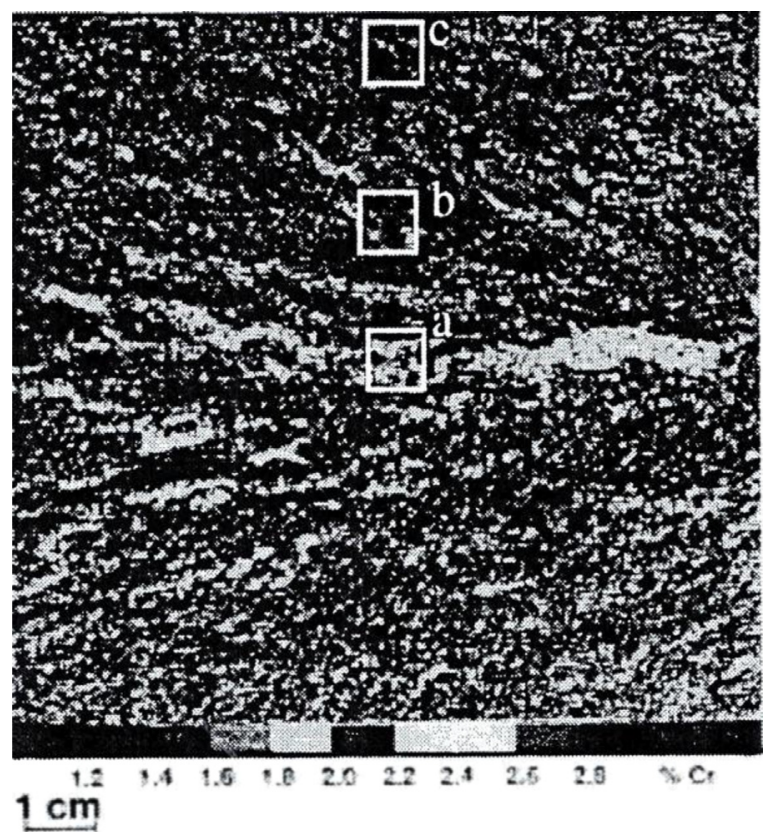

Fig. 22. Cartographie du Cr dans un acier 100Cr6 brut de coulée rotative (\$177) [24]. Les parties claires sont les plus chargées en $\mathrm{Cr}$.

Fig. 22. Cr distribution in as cast 52100 grade on $\Phi 177$ rotative cast bar.

$(\mathrm{Fe}, \mathrm{Cr})_{3} \mathrm{C}$ dont la remise en solution était d'autant plus difficile qu'ils nécessitaient un traitement thermique à plus de $1200^{\circ} \mathrm{C}$ pendant un temps d'autant plus long que les carbures étaient de plus grande taille. Ce traitement thermique de remise en solution (outre son coût) se compliquait, à cause de sa température élevée, d'un risque de brûlure car les zones ségrégées présentaient aussi des enrichissements en éléments $(\mathrm{P}, \mathrm{S})$ conduisant localement à des solidus abaissés. Il convenait donc de minimiser cette présence de carbures primaires dans les structures brutes de coulée, en contrôlant au mieux la fin de solidification au centre du produit.

La figure 22 donne une cartographie du $\mathrm{Cr}$ en zone axiale d'un produit brut de coulée en nuance $100 \mathrm{Cr} 6$ avec la présence des Vés ségrégés. La teneur en $\mathrm{Cr}$ augmente depuis le repère (c) (zone équiaxe) jusqu'au repère (a) (zone axiale ségrégée). Le repère (b) se situe dans un Vé ségrégé. Bien sûr les ségrégations n'étaient pas moindres sur les lingots, mais le double laminage (blooming et train finisseur) et le double réchauffage permettaient d'en minimiser les conséquences sur le produit final.

La figure 23 montre le résultat à $1150{ }^{\circ} \mathrm{C}$ de la solidification de l'acier de base à $1 \% \mathrm{C}$ et $1,5 \% \mathrm{Cr}$ dans la structure austénitique et celle d'une zone ségrégée à teneurs plus élevées en $\mathrm{C}$ et $\mathrm{Cr}$ : austénite plus carbures primaires M3C.

Il convient donc de contrer la mise en place des veines ségrégées si l'on veut minimiser la précipitation de carbures. Les aciéristes s'y sont employés en mettant successivement en œuvre toutes les technologies disponibles: 


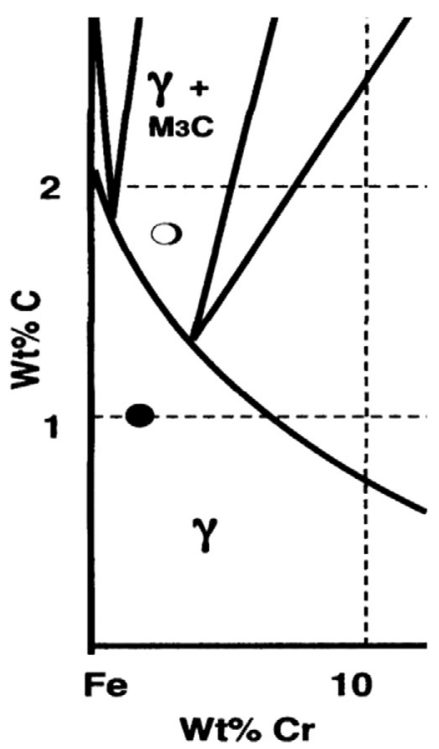

Fig. 23. Représentation schématique des structures d'équilibre à l'état solidifié (section isotherme à $1150^{\circ} \mathrm{C}$ ) d'après [25].

Fig. 23. Equilibrium structures after solidification (isothermal section at $\left.1150^{\circ} \mathrm{C}\right)$.

- en coulant d'abord de très gros produits sur des machines à blooms de grand format $\left(370 \times 480 \mathrm{~mm}^{2}\right.$ chez le Japonais Sanyo Steel) équipées de brasseurs électromagnétiques. Le format coulé permettait de conserver un travail de laminage à chaud suffisant, proche de celui appliqué aux lingots, qui d'expérience assurait une atténuation suffisante des ségrégations;

- en coulant sur machines rotatives (en France chez Vallourec Saint-Saulve et Ascométal Les Dunes) qui amélioraient les structures par rapport aux machines conventionnelles à petits blooms, avec à la suite un forgeage à chaud (machine à forger GFM chez Vallourec Saint-Saulve) pour aboutir à l'ébauche pleine destinée à la tuberie;

- en coulant sur des machines de très gros format équipées de brasseurs électromagnétiques et de réduction en ligne, suivie d'une opération de forgeage comme pour la machine de Timken Faircrest (USA), décrite ci-dessous.

\subsection{La machine à très gros blooms de Timken/ Faircrest}

Cette machine peut être considérée comme emblématique des progrès accomplis par les constructeurs et aciéristes pour maîtriser les problèmes de ségrégations majeures sur produits de forte section.

L'aciérie de Faircrest (USA, Ohio), filiale du fabricant de roulements Timken, s'est équipée en 2014 d'une machine de coulée continue (construite par SMS Concast AG) à trois lignes verticales de blooms présentant les ultimes perfectionnements décrits au fil de cet article qui lui permettent de satisfaire aux hautes exigences des aciers à roulements (santé interne et propreté inclusionnaire). Nous en donnons ci-après les principales caractéristiques:
- le format coulé est de très grandes dimensions. Il s'agit de quasi-lingots : 3 lignes au format $460 \times 610 \mathrm{~mm}^{2}$. C'est probablement la plus grosse machine à blooms au monde. Le format répond à une double exigence de qualité, celle imposée par les aciers à roulements et celle imposée par les demi-produits de forte section, dans laquelle l'usine est aussi spécialisée. Le format coulé permet de conserver en aval, lors de la mise en forme, un taux de corroyage suffisant pour atténuer les ségrégations résiduelles.

- la machine est verticale ce qui permet d'échapper aux désordres internes qu'un décintrage pourrait occasionner. La verticalité fournit un avantage en matière de propreté inclusionnaire puisqu'aucune courbure ne viendra piéger les inclusions non métalliques au cours de leur décantation dans le puits liquide;

- présence de brasseurs électromagnétiques en lingotière (rotatif) et dans le secondaire, ce qui permet d'améliorer les structures de solidification.

- présence d'une réduction mécanique forte en ligne qui par une compression soigneusement calibrée du produit en cours de coulée (pilotage selon le procédé DMSR qui recalcule en temps réel le profil de solidification, voir Sect. 3.5) permet de fermer les veines liquides du cœur pâteux et d'en expulser le liquide ségrégé.

La machine de coulée est alimentée via deux stands de métallurgie secondaire en poche par un four électrique de fusion de $175 \mathrm{t}$.

L'aciérie de Faircrest a une capacité de 900000 t/an dont $10 \%$ d'aciers pour roulements et $30 \%$ de ronds à tubes destinés notamment à l'industrie pétrolière et gazière pour lesquelles les exigences qualitatives sont sévères.

\section{Conclusion partielle relative aux machines de coulée continue conventionnelles}

Les évolutions technologiques que nous venons de décrire à propos de la coulée continue dans sa pénétration de l'ensemble de la sidérurgie, toutes nuances et tous produits confondus, supportées par des développements nombreux en matière de maîtrise des phénomènes physico-chimiques et métallurgiques agissant au cours du processus de solidification, ont finalement abouti à trois grandes familles de machines de coulée répondant à des problématiques assez différentes et conduisant à des conceptions diversifiées pour les machines correspondantes:

- les machines à billettes (aux formats inférieurs à $150 \times 150 \mathrm{~mm}^{2}$ ) destinées aux produits longs (barres, fils, largets...) en nuances courantes (par exemple le rond à béton) pour lesquelles les exigences qualitatives sont en général assez faibles. Les principales qualités exigées de la machine tournent autour de sa productivité et de son coût d'exploitation: vitesse de coulée, disponibilité, simplicité;

- les machines à blooms ou à brames de forte épaisseur pour tôles fortes, très souvent destinées à la coulée des aciers spéciaux de construction. Les exigences métallurgiques sur produits y sont sévères. Le plus souvent le principal problème à résoudre est celui de la maîtrise des ségrégations. Il en résulte pour de telles machines, 
Tableau A1. Coefficients de partage $\mathrm{Cl} / \mathrm{Cs}$ des éléments d'alliage dans le fer liquide vers $1550^{\circ} \mathrm{C}$ d'après [20].

\begin{tabular}{llllll}
\hline Élément & $\mathrm{C}$ & $\mathrm{S}$ & $\mathrm{O}$ & $\mathrm{P}$ & $\mathrm{Cr}, \mathrm{Ni}, \mathrm{Mn}$ \\
\hline $\mathrm{Cl} / \mathrm{Cs}$ & 5 & 20 & 20 & 6,25 & $\approx 1$ \\
\hline
\end{tabular}

une zone de refroidissement secondaire complexe permettant en ligne une maîtrise des ségrégations majeures (brassage électromagnétique secondaire, réduction mécanique en ligne). C'est le secteur sidérurgique où l'on construit encore des machines verticales pour échapper à tous les problèmes posés par la courbure (répartition inclusionnaire, problèmes de décintrage...);

- les machines à brames coulant des produits destinés au $T A B$. Les exigences en matière de qualité de surface du produit coulé y sont extrêmement sévères. Il en découle la nécessité d'une maîtrise très complète du fonctionnement de la tête de machine. Ces nuances étant généralement à bas carbone, les problèmes liés à la ségrégation majeure y sont peu contraignants, ce qui n'empêche pas d'avoir de grandes exigences sur la qualité du soutien dans le secondaire afin d'éviter les défauts internes. Dans ce secteur sidérurgique, les machines, principalement pour des raisons d'encombrement et de productivité, y sont courbes et en général de grand rayon.

En résumé, l'évolution technologique (mécanique, thermique, métallurgique) permet aujourd'hui de couler à peu près toutes les nuances d'aciers aussi bien en produits longs qu'en produits plats. Il a fallu régler les problèmes de qualité de surface, de santé interne et de propreté inclusionnaire, notamment sur les produits plats minces en aciers bas carbone ou inoxydables pour la mise en forme à froid. En produits longs, les aciers à roulements sont coulés en machine en conservant les propriétés d'endurance requises.

\section{Annexe A: La solidification des aciers}

La structure d'un acier solidifié en coulée continue a été décrite dans la figure 1: en périphérie une structure colonnaire à croissance orientée de la peau vers le cœur et au centre une structure équiaxe où les orientations des grains sont aléatoires.

Surfusion de constitution. Contrairement aux métaux purs, la solidification des alliages est, avant tout, pilotée par un phénomène de "surfusion de constitution" (surfusion chimique), résultant du rejet des solutés dans le liquide, à l'interface solide/liquide (voir par exemple Lesoult [20]). Comme c'est le cas dans la plupart des situations métallurgiques courantes, la solubilité des solutés dans le liquide $(\mathrm{Cl})$ est plus élevée que dans le solide $(\mathrm{Cs})$. Ce phénomène est à l'origine de différents types de ségrégation et de modes de croissance du solide, au cours de la solidification. Le coefficient de partage $\mathrm{Cl} / \mathrm{Cs}$ (Tab. A1) mesure le rejet vers le fer liquide de différents solutés: il est élevé pour les métalloïdes et voisin de 1 pour les métalliques, en réalité un peu supérieur à 1 bien qu'il soit difficile de donner des valeurs fiables.

Ainsi, aux vitesses industrielles de refroidissement, l'enrichissement du fer liquide en cours de solidification, est très important en impuretés métalloïdiques, particulièrement pour $\mathrm{S}$ et $\mathrm{O}$, un peu moins pour $\mathrm{P}$ et $\mathrm{C}$ et plus faible pour les éléments d'alliage métalliques. Cependant, les coefficients du tableau A1 ne concernent que les alliages binaires et ne tiennent pas compte des interactions chimiques entre éléments métalliques et métalloïdiques, par exemple Mn-S, Cr-C... qui amplifient les rejets d'éléments métalliques.

Les conséquences de l'enrichissement du liquide au cours de la progression de l'interface solide/liquide vont concerner les ségrégations et les précipitations d'inclusions, ainsi que le mode de croissance du solide.

Ségrégations. Le rejet de soluté enrichit le liquide en éléments métalloïdiques et métalliques, au centre du produit. Compte tenu des dimensions des produits de coulée continue, le travail ultérieur (laminage, traitements thermiques) ne pourra pas homogénéiser le métal. L'enrichissement à cour en impuretés ( $\mathrm{S}, \mathrm{P}, \mathrm{O})$ aura des conséquences possiblement néfastes pour les propriétés d'emploi du produit, en raison de la formation d'inclusions (sulfures, oxydes) au cours du refroidissement et d'hétérogénéités de composition $(\mathrm{C}, \mathrm{P})$.

Interface solide/liquide. Entre la couche solide de structure colonnaire et le liquide, la zone d'interface n'est pas plane. Elle est formée par un squelette de cristaux dendritiques (en forme de branches d'arbres interconnectées) gorgé de liquide constituant une «zone pâteuse»de quelques dizaines de microns à quelques $\mathrm{mm}$, selon la vitesse de refroidissement imposée.

En plus des ségrégations, la progression de la zone pâteuse, repousse au centre du produit les inclusions formées au niveau de la lingotière. Par ailleurs, dans la zone pâteuse de structure peu compacte, le métal solide à haute température où ses propriétés mécaniques sont très basses, pourra se déchirer sous des contraintes diverses (voir le corps du texte) et former des criques internes, elles-mêmes objets de nouvelles ségrégations locales par mouvement de liquide aspiré, créant des défauts internes du produit solide.

Retassures. La variation de volume spécifique du métal à la solidification (contraction) est à l'origine de vides axiaux des produits. En CC, il s'agit de micro-retassures, associées à des inclusions (Fig. 6).

\section{Références}

1. J. Saleil, J. Le Coze, La propreté inclusionnaire des aciers (partie I), Matériaux \& Techniques 103(5), 506, 1 (2015) 
2. M. Durand-Charre, La microstructure des aciers et des fontes, SIRPE, 2003

3. L. Westin, Répartition des inclusions non métalliques dans les aciers au carbone coulés en continu, Congrès international sur la coulée continue (IRSID - The Metals Society), Biarritz, 1976, p. 188

4. J. Choné, Coulée continue de l'acier-outils et défauts, Tech. Ing. M 7811 (2004)

5. W.R. Irving, A. Perkins, Paramètres principaux de contrôle de la qualité des brames, Congrés international sur la coulée continue (IRSID - The Metals Society), Biarritz, 1976, p. 129

6. L. Bäcker, Ph. Gosselin, Quelques aspects de la fabrication des billettes en aciers spéciaux à la coulée continue, Congrès international sur la coulée continue, Biarritz, 1976, p. 180

7. R. Alberny, J.P. Birat, Brassage électromagnétique et qualité des produits, Congrès international sur la coulée continue (IRSID - The Metals Society), Biarritz, 1976, p. 140

8. G. Lesoult, Solidification, macrostructures et qualité des produits, Tech. Ing. M59 (1989)

9. S. Myoshi, Influence des conditions opératoires et des facteurs mécaniques sur la ségrégation centrale des brames, Congrès international sur la coulée continue (IRSID - The Metals Society), Biarritz, 1976, p. 340

10. M. Suzuki, K. Kimura, A. Kawakami, M. Mizutani, Improvement in center segregation of high carbon steel continuous casting blooms, Rev. Métall. 89, 83 (1992), https://doi.org/10.1051/metal/199289010083

11. R. Thome, K. Harste, Principles of Billet Soft-reduction and consequences for continuous casting, ISIJ Int. 46, 1839 (2006)

12. Document Wolf Technology, Synthèse bibliographique (1989-1998)

13. Dillinger Hütte GTS, Notice de présentation, 2017

14. M. Wolf, History of continuous casting, 75th Steel making conference, Toronto, proceedings, 1992, pp. 83-137
15. M.M. Wolf, L'histoire de la coulée continue : Première partie, Rev. Métall. 91, 75 (1994), https://doi.org/10.1051/metal/ 199491010075

16. M.M. Wolf, L'histoire de la coulée continue: Deuxième partie, Rev. Métall. 91, 445 (1994), https://doi.org/10.1051/ metal/199491030445

17. M.M. Wolf, L'histoire de la coulée continue: Troisième partie, Rev. Métall. 91, 589 (1994), https://doi.org/10.1051/ metal/199491040589

18. H. Jacobi, F. Rakoski, High purity in steels as a criterion for materials development, J. Phys IV, colloque UHPM-95, C73, 1995, vol. 5

19. M. Wolf, Strand surface quality and the peritectic reactiona look into the basics, 81st Steelmaking Conference, Toronto, 1998

20. G. Lesoult, Solidification - cristallisation et microstructures, Tech. Ing. M 58, (1986)

21. Y. Nakano, Y. Noguchi, F. Hoshi, Y. Muranaka, Coulée continue d'acier inoxydable, Congrès international sur la coulée continue (IRSID - The Metals Society), Biarritz, 1976, p. 323

22. J. Basilis, G. Jego, Expérience de Decazeville en matière de coulée continue centrifuge, Congrès international sur la coulée continue (IRSID - The Metals Society), Biarritz, 1976, p. 239

23. J. Davené, G. Gay, J. Saleil, Le four électrique à arc. II L'élaboration des aciers inoxydables, Matériaux \& Techniques 102, 301 (2014)

24. P. Benigni, M. Bobadilla, M. Wintz, M. Dylewicz, R. Geoges, Analyse de la mise en solution des carbures dans un acier à roulement 100Cr6, Doc Irsid, octobre 1998

25. Metals Handbook, Metallography, structures and phase diagrams, 8th ed. ASM, Materials Park, OHIO-USA, 1973, vol. 8

26. J. Saleil, J. Le Coze, La coulée continue des aciers - Partie II, Matériaux \& Techniques 106, 504 (2018)

27. J. Saleil, J. Le Coze, La coulée continue des aciers - Partie IV, Matériaux \& Techniques 106, 506 (2018)

Citation de l'article : Jean Saleil, Jean Le Coze, La coulée continue des aciers. Un exemple de développement technique où l'étroite coopération entre métallurgistes, constructeurs et exploitants a été d'une grande fécondité, Matériaux \& Techniques 106, 505 (2018) 